\title{
Zur Mathematik des tierischen Wachstums
}

\author{
III. Testung der Gompertz-Funktion als Wachstumsformel am Beispiel von \\ Siliqua patula (Bivalvia) und Tbunnus tbynnus (Pisces)
}

\author{
F. KRÜGER \\ Biologische Anstalt Helgoland (Zentrale); \\ Palmaille 9, D-2000 Hamburg 50, Bundesrepublik Deutscbland
}

\begin{abstract}
On the mathematics of animal growth. III. Testing the Gompertz function as growth formula using Siliqua patula and Tbunnus thynnus (Pisces). The parameters of the Gompertz function, the Bertalanffy function, and the reciprocal function (Krüger, 1962) are calculated for comparison using growth data obtained by Weymouth et al. $(1930,1931)$ for the razor clam from ten localities and the tuna fish. An new method is employed for the determination of growth parameters under the conditions of linear relations between the power of $e$ and the linear values of size in the Bertalanffy function and its natural logarithm in the Gompertz function. Both equations may therefore be solved by the well known method of linear regressions analysis. The method delivering optimal parameters for the Bertalanffy and the Gompertz function is described in a methodological chapter. Compared to the other functions the Gompertz delivers the best results for the growth curves of the arctic mussels including an inflection point. For curves without inflection points less good results are obtained. Deviations in the numeration of age are compensated in the Gompertz function by the parameter $B$. This parameter represents the difference between the natural logarithms of the upper limit size and the size at the age zero (normally corresponding to the size at birth). The parameter $C$ includes the description of the curvature of the growth curve. A disadvantage of the Gompertz function is, that the upper limit of the equation is very near to the highest numbers evaluated and may be exceeded by real observations. A disadvantage of the reciprocal function is that the calculated inflection point does not correspond to the real inflection point. The results obtained for the relationship between length and weight of tuna fish show that the Gompertz function is exactly compatible with the allometric formula. It delivers for the summing up of the allometric formula the same solution as that reached by the reciprocal function. The three formulas employed are of the same structure, differing only in the use of linear numbers, logarithms or powers of $e$. They deliver good approximations of growth data, but cannot be regarded as exact solutions for the mathematica description of growth curves.
\end{abstract}

\section{EINLEITUNG}

Neben der Pütter-Bertalanffy-Funktion findet immer wieder die sogenannte Gompertz-Funktion in der Literatur Erwähnung. Sie ist seit langem bekannt (Gompertz, 1825) und diente ursprünglich Problemen der Bevölkerungsstatistik.

Bei dem Bemühen, ihr umfangreiches Zahlenmaterial über das Wachstum der 
Muschel Siliqua patula von der Westküste Nord-Amerikas für einen exakten Vergleich mathematisch zu beschreiben, stießen Weymouth et al. $(1930,1931)$ auf die Schwierigkeit, daß in den Wachstumskurven der Muscheln aus Alaska ein Wendepunkt auftritt, den die Bertalanffy-Funktion nicht wiederzugeben vermag. Theoretische Uberlegungen führten dann die Autoren zur Gompertz-Funktion als einer Möglichkeit zur Darstellung von Wachstumskurven mit Wendepunkt. Der Auswertung ihres großen Zahlenmaterials waren aber mit den damals zur Verfügung stehenden rechnerischen Hilfsmitteln sehr enge Grenzen gesetzt.

Nachdem mit modernen Elektronen-Rechnern auch die Auswertung komplizierterer Exponentialfunktionen keine Schwierigkeiten mehr bereitet, erschien es mir wünschenswert, die von den Autoren gezogenen Schlüsse an ihrem eigenen Zahlenmaterial zu prüfen, vor allem, da auch heute noch ihre Veröffentlichung als Grundlage für die Anwendung der Gompertz-Funktion als Wachstumsfunktion immer wieder zitiert wird.

Die numerische Auswertung aller von Weymouth und Mitarbeitern veröffentlichten Wachstumsreihen führte zu interessanten Einsichten in die Eigenschaften der Gompertz-Funktion. Gleichzeitig machte sie deutlich, daß die Daten für das Längen-Wachstum von Siliqua patula nicht als ein ganz ideales Beispiel für die Testung einer Wachstums-Funktion angesehen werden können.

In der Zwischenzeit hatten Zucker \& Zucker (1941) eine neue Funktion vorgeschlagen, die ebenfalls einen Wendepunkt enthält. Ich habe sie in etwas veränderter Form mit gutem Erfolg auf eine Reihe von Beispielen anwenden können (Krüger, 1962, 1973). Sie wird im folgenden als Reziprokfunktion bezeichnet.

Da bei der Mehrzahl der von Weymouth et al. veröffentlichten Zahlenreihen der Wendepunkt offensichtlich in der Nähe des Anfangswertes zu suchen ist, erschien ihre Auswertung auf der Basis der Pütter-Bertalanffy-Funktion nicht aussichtslos, was die Ergebnisse auch bestätigten. Die auf diese Weise durchgeführte Anwendung von drei Funktionen auf das gleiche Zahlenmaterial ließ die Möglichkeit aber auch die Grenzen einer mathematischen Erfassung von Wachstumsvorgängen besonders deurlich hervortreten.

Weymouth et al. konnten für das einzige von ihnen veröffentlichte Beispiel - nämlich für das Wachstum der Muscheln aus der Hallo Bay - keine überzeugende Lösung geben, da sie eine befriedigende Annäherung an die gegebene Zahlenreihe nur durch eine Unterteilung in zwei Abschnitte erreichen konnten. Jede Unterteilung eines zusammenhängenden Kurvenverlaufs stellt aber geringere Anforderungen an die spezifische Eignung einer Funktion zur Beschreibung eines quantitativen Zusammenhangs. Es muß daher stets das Bestreben einer Wachstumsmathematik sein, mit ihren Funktionen möglichst weite Spannen - sowohl hinsichtlich der Zeit wie auch der Dimension - mit einheitlichen Parametern zu erfassen.

Unterteilungen eines zusammenhängenden Wachstumsablaufs erscheinen höchsiens gerechtfertigt, wenn sie mit biologisch deutbaren Grenzen zusammenfallen, wie etwa dem Abschluß der Embryonal- oder Larval-Entwicklung. Bei den vorliegenden Auswertungen erfolgten daher grundsätzlich keine Unterteilungen, die sich auch nicht als erforderlich erwiesen.

Meßfehler dürften bei der Bestimmung der Muschellängen kaum aufgetreten sein, 
aber trotz der großen Zahl gemessener Individuen enthält eine Reihe der Tabellen vor allem bei den höheren Altersgruppen - unregelmäßige Wachstumsraten mit zum Teil rückläufigen Werten. Durch die Lage am Ende der Zahlenreihen beeinträchtigen solche irregulären Werte die Parameter-Bestimmung auf der Basis der Regressionsberechnung.

Die Bestimmung des Alters der Muscheln ermöglichen die jährlichen Zuwachsringe in den Schalen. Wenn diese Jahresringe - ebenso wie von Weymouth et al. - den rechnerischen Auswertungen zugrunde gelegt wurden, ist zu berücksichtigen, daß sie nur grobe Alters-Schätzungen bieten. Nach Angabe der Autoren sind sie vermutlich um ein halbes Jahr zu hoch.

Da verschiedene Erfahrungen erkennen ließen, daß die von mir bislang angewandte Bestimmung der Parameter der Bertalanffy- und der Gompertz-Funktion nicht zu optimalen Ergebnissen führt, da ihre Grundlage nicht die Variable y selbst, sondern ihr Abstand vom Maximalwert bildet, wandte ich in dieser Arbeit ein Verfahren an, das direkt die Beziehung von y bzw. In y zu dem Alterswert herstellt. Es soll im folgenden kurz erläutert werden.

\section{METHODIK}

Aus der schon von Pütter (1920) vermuteten quantitativen Beziehung zwischen auf- und abbauenden Prozessen beim Wachstum leitete v. Bertalanffy (1934) die Formulierung $a b$ :

$$
l \chi=L_{\infty}-\left(L_{\infty}-L_{0}\right) \cdot \mathrm{e}^{-K \cdot \chi}
$$

In ihr stellt $l \chi$ die Länge im Alter $\chi$ dar. (Aus bestimmten Gründen benutze ich für Zeitwerte allgemein griechische Symbole, und zwar $\chi$ für das Geburtsalter und $\tau$ für allgemeine Zeitwerte.) $L_{\infty}, L_{0}$ und $K$ stellen die Parameter dar, die aus den Meßwerten zu errechnen sind und den Verlauf der Wachstumskurve charakterisieren.

Gleichung (1) beschreibt eine lineare Beziehung zwischen $l \chi$ und $\mathrm{e}^{-K} \cdot \overline{\text { und }}$ ist daher für die Parameterbestimmung auf dem Wege der linearen Regressionsberechnung zu lösen. In der Literatur findet man hierfür die logarithmierte Form der Bertalanffy-Funktion angegeben. Sie hat den oben erwähnten Nachteil, nicht $l_{\chi}$ selbst, sondern $\left(L_{\infty}-l_{\chi}\right)$ als Funktion von $\chi$ darzustellen. Diesen Fehler vermeidet Gl. (1), aus der sich $l \chi$ als Funktion von $\mathrm{e}^{-K \cdot \chi}$ und - bei konstantem $K$ - damit direkt von $\chi$ ergibt.

Zur Durchführung der Parameterbestimmung setzt man zunächst für den Parameter $K$ einen Schätzwert ein, der zur Bestimmung der Werte von $\mathrm{e}^{-K \cdot \gamma}$ bei der Regressionsberechnung erforderlich ist und zu den zugehörigen Werten von $L_{\infty}$ und $L_{0}$ führt. Diese sind allerdings zunächst nicht von Interesse, sondern nur die im Verlauf der Regressionsberechnung anfallende Standardabweichung ( $s$ ) bzw. der Korrelationskoeffizient $(r)$. Man variiert nun für die iterative Berechnung den Wert von $K$ solange, bis die Strauungsmaße eine optimale Annäherung an die Meßdaten ergeben. Durch Interpolation kann man die Zahl der iterativen Schritte vermindern. Einen ersten, sehr guten Näherungswert für $K$ kann man für die Bertalanffy-Funktion aus der Ford-Walford-Beziehung Gl. (8) errechnen. 
Von großem Einfluß auf die Ermittlung der Parameter-Werte ist die Definition der optimalen Näherung. Bei der Auswertung der Bertalanffy-Funktion in der besprochenen Weise erhält man die Standard-Abweichung der linearen Werte $(s)$, die die Summe der quadrierten linearen Differenzen zwischen gemessenen und berechneten Werten zum Minimum macht. Für Wachstums-Untersuchungen erscheint es mir aber günstiger, eine relative Standardabweichung zu benutzen, die für Organismen unterschiedlicher Größe vergleichbare Werte liefert.

Die relative Standardabweichung ergibt sich aus der Summe der Differenzen der Logarithmen der einzelnen Werte-Paare; ich bezeichne sie mit $s_{\log }$ :

$$
s_{\log }= \pm \sqrt{\frac{(\log y-\log \hat{y})^{2}}{n-3}}
$$

Es bedeuten: $y=$ Meßwert; $\hat{y}=$ errechneter Wert; $\mathbf{n}=$ Anzahl der Wertepaare.

Die logarithmische Standardabweichung läßt sich leicht in einen besser durchschaubaren Prozentwert umrechnen:

$$
s_{0 \%}=\left(100 \cdot \text { num } s_{10 g}\right)-100
$$

In den Tabellen gebe ich nicht den Wert des Parameters $\left(L_{\infty}-L_{0}\right)$ der BertalanffyFunktion an, sondern den Zeitwert $\tau_{0}$, der den Zeitpunkt kennzeichnet, zu dem rechnerisch der Organismus die Dimension Null hätte. $\tau_{0}$ stammt aus der erstmals von Bagenal (1955) vorgeschlagenen Formulierung der Bertalanffy-Funktion

$$
l_{\chi}=L_{\infty} \cdot\left(1-\mathrm{e}^{-K\left(\chi-\tau_{0}\right)}\right)
$$

$\tau_{0}$ errechnet sich aus den Parametern von Gl. (1) nach:

$$
\tau_{0}=\frac{\ln L_{\infty}-\ln \left(L_{\infty}-L_{0}\right)}{K}
$$

Die Gompertz-Funktion hat die Gestalt:

$$
\mathrm{y}_{\chi}=A \cdot \mathrm{e}^{-B \cdot \mathrm{e}^{-C \cdot \chi}}
$$

Sie ergibt durch Logarithmierung:

$$
\ln y_{\chi}=\ln A-B \cdot \mathrm{e}^{-C \cdot \chi}
$$

Man erkennt leicht, daß die logarithmierte Gompertz-Funktion den gleichen Aufbau zeigt wie die Bertalanffy-Funktion Gl. (1), nur daß anstelle der linearen Zahlen deren natürliche Logarithmen stehen. Die Gompertz-Funktion beschreibt also eine lineare Beziehung zwischer، In $y_{\chi}$ und $\mathrm{e}^{-\mathrm{C}} \cdot \chi$. Daher können wir, wenn wir für C eine geeignete Zahl und die natirlichen Logarithmen der Meßwerte einsetzen, auf dem Wege der linearen Regressiorsberechnung die Werte der zugehörigen Parameter $A\left(=\ln Y_{\infty}\right)$ und $B$ ermitteln. Als Standardabweichung, die für die Auffindung des optimalen Wertes von $C$ auf dem Wege der iterativen Berechnung von besonderer Bedeutung ist, erhalten wir verständlicherweise die Standardabweichung der natürlichen Logarithmen, die nach Gl. (3) leicht in die prozentuale Standardabweichung umzuwandeln ist.

Die Ermittlung der optimalen Parameter auf dem Wege der iterativen Berechnung bereitet bei der Rechengeschwindigkeit der modernen Elektronenrechner keinen erheblichen Zeitaufwand. Trotzdem ist es eine Erleichterung, wenn man einen Nähe- 
rungswert für den einzusetzenden Parameter kennt. Für die Bertalanffy- und die Gompertz-Funktion besteht hierfür ein relativ leicht durchzuführendes Verfahren.

Eliminiert man in der Bertalanffy-Funktion in der Formulierung für zwei aufeinanderfolgende Altersstufen $\left(\chi\right.$ und $[\chi+1]$ ) der Gl. (1) den Parameter $\left(L_{\infty}-L_{0}\right)$, so kommt man für die Beziehung zwischen den beiden Längenwerten zu der seit Ford (1933) und Walford (1946) bekannten Formel:

$$
l_{(\chi+1)}=a+b \cdot l_{\chi}
$$

Aus den beiden Parametern dieser Gleichung - $a$ und $b$ - errechnen sich die Bertalanffy-Parameter nach:

$$
K=-\ln b \quad \text { und } \quad L_{\infty}=\frac{a}{1-b}
$$

Durch die formale Übereinstimmung der logarithmierten Gompertz- mit der Bertalanffy-Funktion ist leicht verständlich, daß sich aus ihr die Beziehung ableitet (Bagenal, 1955b):

$$
\ln y_{(x+1)}=a+b \cdot \ln y_{\chi}
$$

Zur Ermittlung der Parameter setzt man in der Ford-Walford-Auswertung anstelle der natürlichen Zahlen deren Logarithmen ein. Es ist dann:

$$
C=-\ln b \quad \text { und } \quad \ln A=\ln Y_{\infty}=\frac{a}{1-b}
$$

Zwei Algol-Computer-Programme zur Ermittlung der Gompertz-Parameter für das menschliche Embryonalwachstum veröffentlichten neuerdings Scharf \& Peil (1975).

Für die Reziprokfunktion benutze ich die Schreibung:

$$
\mathrm{y}_{\chi}=\frac{Y_{\max }}{\frac{1}{N \chi^{+\zeta}}}
$$

Die Parameter sind die rechnerische Maximalgröße $Y_{m a x}$, die "Geschwindigkeitskonstante" $N$ und der additive Alterswert $\xi$.

Durch Logarithmierung geht auch diese Funktion in die Gleichung einer Geraden über:

$$
\log y_{\chi}=\log Y_{\max }-\frac{1}{\chi+\zeta} \log N
$$

Die Bestimmung der Parameterwerte und der Standardabweichung auf dem Wege der linearen Regressionsberechnung habe ich bereits früher beschrieben (Krüger, 1973).

Übereinstimmend habe ich bei den drei angewandten Funktionen das Geburtsalter mit $\chi$ bezeichnet, es stellt also das in den Tabellen angegebene Alter dar. Die zugehörige Größe habe ich dementsprechend mit $\mathrm{y}_{\chi}$ und bei der Bertalanffy-Funktion, die nur auf Längenwerte anzuwenden ist, mit $l_{\chi}$ bezeichnet. Für das additive Alter in der von Bagenal (1955a) und später von Beverton \& Holt (1957) modifizierten Bertalanffy-Funktion benutze ich $\tau_{0}$ als Symbol und für das additive Alter in der Reziprokfunktion das Symbol $\xi$.

Die Mehrzahl der von Weymouth et al (1930, 1931) angegebenen Muschel- 
Iängen stellen Medianwerte dar. Für die höheren Altersgruppen, für die nicht genügend Material vorlag, veröffentlichten die Autoren Mittelwerte, die aber zum Teil offensichtlich unzuverlässig sind. Aus diesem Grund wurden nur die besser gesicherten Medianwerte den Parameter-Bestimmungen zugrunde gelegt und die in den Tabellen gekennzeichneten Mittelwerte grundsätzlich außer Betracht gelassen. Durch Extrapolation über den ausgewerteten Bereich hinaus wurde aber geprüf, wieweit sie den Erwartungswerten entsprachen.

Wie schon oben erwähnt, stellen die in den Tabellen angegebenen Alterswerte nur grobe Näherungen dar. Die Bertalanffy-Funktion, in Form der Gleichung (4), und die Reziprokfunktion können durch das in ihnen auftretende additive Alter solche Abweichungen vom wirklichen Wert in leicht verständlicher Weise ausgleichen. Die Funktionen führen jedoch nicht zu übereinstimmenden Ergebnissen für den Zeitpunkt der Geburt; er hängt vom Verlauf der von den Funktionen beschriebenen Kurven ab.

Auch die Gompertz-Funktion hat die Eigenschaft, fehlerhafte Altersangaben auszugleichen. Zum Geburtstermin kann man kommen, wenn man aus der Funktion das Alter von 0,1 mm langen Muscheln berechnet, wie von Weymouth \& McMillin (1930) für die neugeborenen Muscheln angegeben.

\section{DAS WACHSTUM DER MUSCHELN AUS DER HALLO BAY}

Das Wachstum der Muscheln aus der Hallo Bay soll gesondert behandelt werden, da es das einzige Beispiel ist, für das die Autoren eine numerische Auswertung veröffentlicht haben. In der graphischen Darstellung der logarithmischen Wachstumsraten glaubten sie eine Unstetigkeit bei der Altersstufe 7 finden zu können und unterteilten aus diesem Grund an dieser Stelle die Wachstumsreihe und errechneten gesonderte Parameter für die ersten 7 und für die folgenden 12 Altersstufen. Ich werde mich bei der Besprechung auf die erste Gruppe beschränken. Als prozentuale Standardabweichung ergibt sich für ihre Parameterwerte 3,16\%. Mit den von mir ermittelten Parametern $\left[A\left(\mathrm{Y}_{\infty}\right)=13,7309 ; B=7,36902 ; C=0,6977\right]$ komme ich auf eine Standardabweichung von nur 1,77\%. Auch hinsichtlich der linearen Annäherung, die vermutlich Weymouth et al. anstrebten, erwiesen sich die angegebenen Parameter als uiberlegen.

Angesichts der Schwierigkeit, die noch bis vor kurzem die Parameterbestimmung der Wachstumsfunktionen bereitete, erschien es mir notwendig zu prüfen, ob es möglich ist, die Gesamtheit der hinreichend gesicherten Daten der Altersstufen 1-15 mit einem einheitlichen Satz von Parametern wiederzugeben. Als optimale Lösung ergab sich hierbei eine relative Standardabweichung von $4,23 \%$, was bei einer so langen Datenreihe und so erheblichen Größenunterschieden als durchaus befriedigend angesehen werden kann. Die zugehörigen Parameterwerte sind: $A=14,79 ; B=7,2307$; $C=0,6570$. Demgegenüber schneidet die Reziprokfunktion, die optimal eine Näherung von $9,36 \%$ ergibt, sehr schlecht ab.

Trotzdem kann man die Gompertz-Funktion nicht als eine ganz ideale Lösung betrachten, da der Wert von $A$, der den asymptotischen Grenzwert der Funk- 
Tabelle 1

"Hallo Bay"-Einzelwerte von Siliqua patula der drei berücksichtigten Funktionen und ihre prozentualen Abweichungen von den Meßwerten. Die den Zahlen zugrunde liegenden Parameter sind aus den besser gesicherten Medianwerten der Altersstufen 1-15 errechnet. Die Werte der Altersstufen 16-19 wurden aus diesen Parametern extrapoliert

\begin{tabular}{|c|c|c|c|c|c|c|c|}
\hline $\begin{array}{c}\text { Alter } \\
\text { (Jahre) }\end{array}$ & $\begin{array}{l}\text { Meßwert } \\
(\mathrm{cm})\end{array}$ & $\begin{array}{l}\text { Gompertz } \\
(\mathrm{cm})\end{array}$ & $\begin{array}{c}\text {-Funktion } \\
(\% / 0 \mathrm{Abw} .)\end{array}$ & $\begin{array}{l}\text { Bertalanff } \\
\quad(\mathrm{cm})\end{array}$ & $\begin{array}{c}\text { fy-Funktion } \\
(\% \mathrm{Abw} .)\end{array}$ & $\begin{array}{l}\text { Reziprol } \\
(\mathrm{cm})\end{array}$ & $\begin{array}{l}\text { kfunktion } \\
(\% \% \mathrm{Abw} .)\end{array}$ \\
\hline 1 & 0,34 & 0,348 & $+2,48$ & $-2,755$ & $-910,4$ & 0,330 & $-3,08$ \\
\hline 2 & 2,25 & 2,119 & $-5,84$ & 2,228 & $-0,97$ & 2,695 & $+19,79$ \\
\hline 3 & 5,42 & 5,401 & $-0,35$ & 5,874 & $+8,39$ & 5,456 & $+0,66$ \\
\hline 4 & 8,60 & 8,774 & $+2,02$ & 8,542 & $-0,67$ & 7,769 & $-9,66$ \\
\hline 5 & 10,96 & 11,283 & $+2,95$ & 10,495 & $-4,25$ & 9,607 & $-12,35$ \\
\hline 6 & 12,37 & 12,854 & $+3,91$ & 11,923 & $-3,61$ & 11,069 & $-10,52$ \\
\hline 7 & 13,17 & 13,753 & $+4,43$ & 12,968 & $-1,53$ & 12,249 & $-6,99$ \\
\hline 8 & 13,65 & 14,243 & $+4,35$ & 13,733 & $+0,61$ & 13,216 & $-3,18$ \\
\hline 9 & 14,06 & 14,504 & $+3,16$ & 14,292 & $+1,65$ & 14,021 & $+0,28$ \\
\hline 10 & 14,44 & 14,642 & $+1,40$ & 14,702 & $+1,81$ & 14,700 & $+1,80$ \\
\hline 11 & 14,75 & 14,713 & $-0,25$ & 15,001 & $+1,70$ & 15,280 & $+3,59$ \\
\hline 12 & 15,08 & 14,751 & $-2,18$ & 15,220 & $+0,93$ & 15,781 & $+4,65$ \\
\hline 13 & 15,38 & 14,770 & $-3,97$ & 15,381 & $-0,004$ & 16,218 & $+5,45$ \\
\hline 14 & 15,50 & 14,780 & $-4,65$ & 15,498 & $-0,01$ & 16,602 & $+7,11$ \\
\hline 15 & 15,80 & 14,785 & $-6,42$ & 15,584 & $-1,37$ & 16,942 & $+7,23$ \\
\hline 16 & 15,61 & 14,788 & $-5,27$ & 15,647 & $+0,24$ & 17,246 & $+10,48$ \\
\hline 17 & 15,74 & 14,789 & $-6,04$ & 15,693 & $-0,30$ & 17,518 & $+11,30$ \\
\hline 18 & 16,31 & 14,790 & $-9,32$ & 15,726 & $-3,58$ & 17,763 & $+8,91$ \\
\hline 19 & 16,74 & 14,790 & $-11,65$ & 15,751 & $-5,91$ & 17,986 & $+7,45$ \\
\hline & & \multicolumn{2}{|c|}{$\begin{array}{l}A=14,79075 \mathrm{~cm} \\
B=7,23074 \\
C=0,6570 \\
s^{0}=4,23\end{array}$} & \multicolumn{2}{|c|}{$\begin{aligned} L \infty & =15,818 \mathrm{~cm} \\
K & =0,3124 \\
\tau_{0} & =1,514\end{aligned}$} & \multicolumn{2}{|c|}{$\begin{aligned} L \infty & =22,512 \mathrm{~cm} \\
\log N & =1,85285 \\
\xi & =0,01 \\
s^{0} \% & =9,36\end{aligned}$} \\
\hline
\end{tabular}

tion für das Alter $\infty$ darstellt, noch unterhalb der höchsten ausgewerteten Zahl $(15,80)$ liegt und letzterer Wert nicht einmal ein Maximum für das Wachstum der Muscheln bildet. Es steht also dieser mathematische Grenzwert im Widerspruch zu biologischen Gegebenheiten. Diese Bedenken treffen auch auf die anderen Meßreihen von Weymouth et al. zu.

Eine unerwartet gute Näherung an die Daten nach dem Wendepunkt bei 3 Jahren liefert die Pütter-Bertalanffy-Funktion, wie die Tabelle 1 zeigt, in der ich für dieses eine Beispiel eine ausführliche Nebeneinanderstellung der errechneten Werte der drei Funktionen gebe. Die Pütter-Bertalanffy-Funktion liefert vor dem Wendepunkt erwartungsgemäß vollkommen abwcichende Zahlen und für die erste Altersstufe sogar einen negativen Wert. Dieser Umstand schließt eine sinnvolle Anwendung der relativen Standardabweichung als Streuungsmaß naturgemäß aus, sie ist daher auch nicht angegeben.

$\mathrm{Da}$ anscheinend im Anfangsteil der Datenreihe die Schwierigkeiten für die mathematische Wiedergabe liegen, unternahm ich den Versuch, die Parameter unter 
Auslassung des Anfangswertes - also nur für die Altersstufen 2-15 - zu errechnen. Für die Gompertz-Funktion ergab sich hierdurch eine Verbesserung der StandardAbweichung auf 3,17\%. Für die Pütter-Bertalanffy-Funktion lag mit 3,25\% die Standardabweichung nur unwesentlich höher, obwohl auch diese Reihe noch den bei Altersstufe 3 liegenden Wendepunkt enthält. Zwar wesentlich besser, aber schlechter als bei den beiden vorgenannten Funktionen war mit 4,01\% die Standardabweichung der Reziprokfunktion.

Es zeigte sich, daß allgemein bei den Muscheln von Alaska mit einem spät liegenden Wendepunkt die Fortlassung des Anfangswertes bessere Näherungen an die ïbrigen Daten erbrachte. Die auf diese Weise errechneten Parameter sind im unteren Teil der Tabelle 2 angegeben.

Die Anfangswerte nicht $z u$ berïcksichtigen erscheint aus dem Grund vertretbar, da die Schalen von Siliqua patula im Verlauf ihres Wachstums erhebliche Proportionsänderungen erfahren. Sie ergeben für die Jugendstadien erhebliche Abweichungen von einem normalen Kurvenverlauf, wie er von den Funktionen beschrieben wird.

Gegen eine begründete Auslassung einzelner Daten kann man bei Wachstumsauswertungen keine ernstlichen Einwände erheben, da sich bei zutreffenden Daten und zutreffender mathematischer Formulierung aus jedem beliebigen Kurvenstïck theoretisch die gleichen Parameter ableiten lassen. Ich halte es für günstiger - unter Verzicht auf einen einzelnen Wert - die ganze übrige Wachstumsspanne mit einheitlichen Parametern zu erfassen, als sie durch zwei unterschiedliche Sätze von Parametern zu beschreiben.

\section{VERGLEICH DES WACHSTUMS DER MUSCHELN UNTERSCHIEDLICHER HERKUNFT}

Weymouth et al. (1930, 1931) begnügten sich nicht mit der Erfassung des Wachstums von Siliqua patula von einem Fundort, sondern verarbeiteten Material von insgesamt 10 ökologisch unterschiedenen Biotopen an der Westküste von Nordamerika zwischen Californien und Alaska. Die von ihnen angegebene geographische Breite ist in Tabelle 2 aufgenommen.

Eine Übersicht über die aus den Wachstumsdaten der 10 Fundorte ermittelten Parameter gibt Tabelle 2. Es zeigt sich, daß sich durchweg die Zahlenreihen für die Medianwerte in befriedigender bis guter Näherung durch einheitliche Parameter wiedergeben lassen, wenn man den Streubereich der Meßwerte berücksichtigt. Wenn auch die angegebenen Pàrameter nicht alle Einzelheiten der Zahlenreihen zum Ausdruck bringen, sollen sie doch als Grundlage eines Vergleichs dienen.

Begonnen werden soll mit der Güte der Annäherung der Funktionen an die Tabellenwerte. Sie erfolgt auf der Grundlage der prozentualen Standardabweichung. Die Gompertz-Funktion lieferte mit nur einer Ausnahme bei den einen Wendepunkt einschließenden Daten der Muscheln aus der Arktis, wenn man die Parameter der verkürzten Reihen wählt, eine bessere Wiedergabe der Wachstumskurven als die beiden anderen Funktionen. Die Reziprokfunktion schneidet bei ihnen recht 


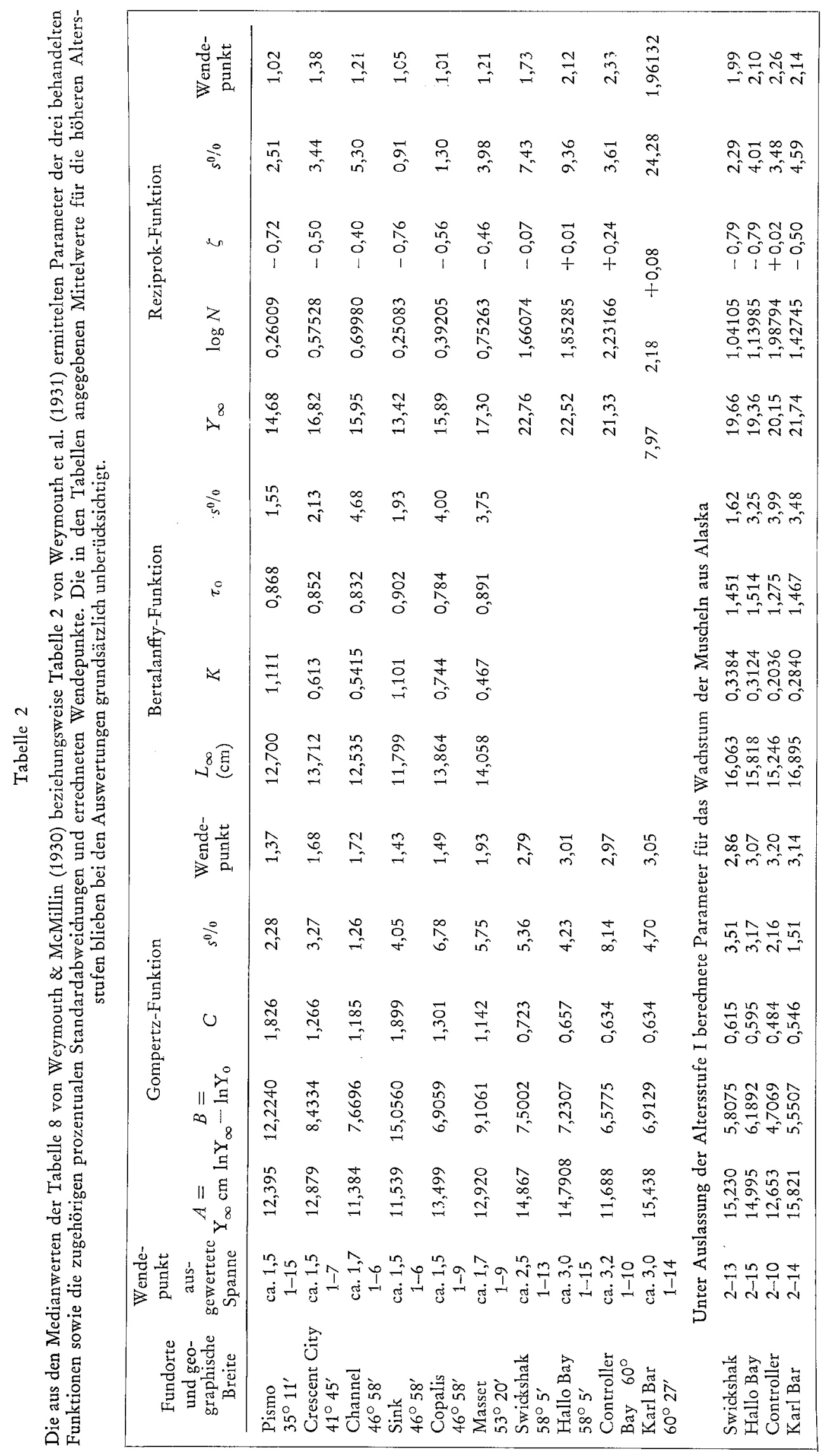


schlecht ab, denn ihre Näherung wird in drei Fällen sogar von der Pütter-BertalanffyFunktion übertroffen.

Bei den Datenreihen ohne Wendepunkt erweist sich die Gompertz-Funktion weniger geeignet und liefert nur in einem Fall das beste Ergebnis, während die Pütter-Bertalanffy-Funktion in 3 Fällen und die Reziprokfunktion in 2 Fällen die beiden anderen Funktionen übertreffen.

Es ergibt sich also, daß alle drei Funktionen geeignet erscheinen, die vorliegenden Wachstumsdaten näherungsweise wiederzugeben. Wenn auch die Reziprokfunktion bei dem Vergleich der Standardabweichungen nicht allzugut abschneidet, so kann man sie trotzdem nicht als ungeeignet bezeichnen und keiner der Funktionen einen eindeutigen Vorrang zuerkennen. Den ungünstigen Ergebnissen der Reziprokfunktion stehen die häufig besseren Näherungen der Gompertz-Funktion gegenüber. Es besteht hier ein gewisser Antagonismus zwischen diesen beiden Wachstumsformeln. Das ist leicht verständlich, da beide Funktionen unterschiedliche Kurven beschreiben und je näher die Meßwerte bei der einen Kurve liegen, um so weiter entfernen sie sich von der anderen. Durch die besonderen Bedingungen, die beim Linearwachstum von Siliqua patula vorliegen, entsprechen die Daten besser der Gompertz-Funktion als der Reziprokfunktion. Es ist aber nicht zulässig, dieses Ergebnis zu verallgemeinern. Das Verhältnis der Streuungswerte der Pütter-Bertalanffy-Funktion entspricht etwa denen der Reziprokfunktion.

Im folgenden soll nun versucht werden, die Parameterwerte der 3 berücksichtigten Funktionen in Beziehung zu den Gegebenheiten ihrer Umwelt zu setzen.

Der in den drei Funktionen enthaltene Maximalwert ist die Größe, der sich der Organismus bei unbegrenzter Lebensdauer asymptotisch nähern würde. Diese Definition macht den rein formalen Charakter dieses Parameters deutlich. Der Wert der Maximalgröße hängt von der Wachstums-Formulierung $a b$ und macht daher jede biologische Interpretation fraglich.

Für die Gompertz-Funktion ergaben sich in 8 Fällen für die Maximalgröße Werte, die noch unterhalb des höchsten Wertes der zugrunde gelegten Zahlenreihe lagen. In den beiden anderen Fällen lag er sehr kurz darüber.

Das in Tabelle 1 wiedergegebene Beispiel der Muscheln von der Hallo Bay zeigt, daß sich durch die frühe Annäherung an den Grenzwert die theoretischen Werte etwa ab Altersstufe 12 - nur noch geringfügig ändern, während die Meßwerte noch erheblich ansteigen. Die relativ geringen Zuwachsraten der älteren Jahresklassen bedingen, daß der Bezug auf den nahezu erreichten oder auch überschrittenen Endwert nur zu geringen Erhöhungen der prozentualen Standardabweichung beitragen. Bei der Extrapolation in den nicht ausgewerteten Bereichen nehmen die Ảbweichungen aber unzulässig stark zu.

Trotz der zumeist befriedigenden Annäherung an die Meßdaten kann man die Gompertz-Funktion daher durch die sehr niedrigen Maximalwerte nicht als eine ganz ideale Lösung ansehen.

Der Maximalwert der Pütter-Bertalanffy-Funktion liegt allgemein höher als der entsprechende Parameter der Gompertz-Funktion. Das Beispiel der Tabelle 1 macht diesen Unterschied dadurch deutlich, daß im extrapolierten Bereich die Annäherung an die Meßwerte sehr gut ist. Aber auch in diesem Fall liegt der Maximalwert unter- 
halb der Zahlen für die Altersstufen 18 und 19, stellt also auch keinen wirklichen oberen Grenzwert für das Wachstum dar.

Der Maximalwert der Reziprokfunktion liegt in allen Fällen - zum Teil erheblich - über den höchsten Meßwerten und läuft daher kaum Gefahr, von realen Messungen übertroffen zu werden. Im Beispiel der Muscheln von der "Hallo Bay" liegt der Maximalwert bei $22,5 \mathrm{~cm}$ gegenüber $15,8 \mathrm{~cm}$ beziehungsweise $15,0 \mathrm{~cm}$ bei den beiden anderen Funktionen. Die Maximalwerte der Reziprokfunktion überschreiten ohne Ausnahme die Mittelwerte der alten Muscheln, die bei der Auswertung nicht berücksichtigt wurden. Für die Gompertz-Funktion triff das nur für die Muscheln von den Fundorten "Controller" und "Karl Bar" zu. Für die Pütter-Bertalanffy-Funktion liegen nur für "Sink" und "Copalis" die Maximalwerte unterhalb der höchsten ausgewerteten Zahlen.

Eine Betrachtung der Tabelle 2 läßt erkennen, daß die Maximalwerte aller drei Funktionen eine zwar nicht strenge, aber doch deutlich zutage tretende Tendenz zu höheren Werten bei den nördlichen Populationen erkennen lassen. Es steht das in Ubereinstimmung mit der Entwicklung größerer Tierarten in den kalten Bereichen der Erde. Es bleibt hierbei offen, ob der auch an dem gesammelten Material zutage tretende Unterschied lediglich eine Temperaturwirkung darstellt oder ob lokale Rassen vorliegen. Da die bei "Copalis", "Sink" und "Channel" in dichter Nachbarschaft lebenden Muscheln unterschiedliche Maximalwerte liefern, scheinen auch die Ernährungsbedingungen einen Einfluß zu haben.

\section{DER AUSGLEICH DER ALTERSWERTE}

Im Gegensatz zu Länge und Gewicht, die mit Zuverlässigkeit gemessen werden, stellen die Altersangaben fast ausnahmslos nur Näherungen dar. Bei freilebenden Tieren ist der genaue Termin der Geburt bzw. der Metamorphose in der Regel unbekannt und eine exakte Altersangabe daher nicht möglich.

Die drei in dieser Untersuchung berücksichtigten Funktionen besitzen nun die Eigenschaft, daß sie von systematischen Fehlern der Altersangaben unabhängig sind. Bei der Reziprok- und der Bertalanffy-Funktion beruht diese Korrektur auf den additiven Alterswerten $-\zeta$ bzw. $\tau_{0}-$, die die Altersangaben in entsprechender Weise anpassen. Mathematisch stellen diese additiven Alterswerte ein pränatales Alter dar, jedoch dürfte biologisch diese Interpretation im allgemeinen nicht zutreffend sein.

$\mathrm{Da}$ gerade bei den vorliegenden Beispielen die auf den Jahresringen basierenden Alterswerte nach Angabe der Autoren vermutlich um etwa ein halbes Jahre zu hoch sind, führte ich zur Orientierung die Parameter-Berechnungen mit um 0,5 verminderten Altersangaben durch. Wie erwartet, ergaben sich bei der Reziprok- und der Bertalanffy-Funktion für $\zeta$ und $\tau_{0}$ um 0,5 verminderte Werte, während die beiden anderen Parameter sowie dic prozentuale Standardabweichung durch die Änderung der AltersSkala nicht beeinflußt wurden.

Das gleiche Verhalten zeigt auch die Gompertz-Funktion, wenn auch nicht in so durchsichtiger Weise. Die Verschiebung der Alters-Skala läßt die Parameter $A$ und $C$ sowie die prozentuale Standardabweichung unverändert; die Anpassung erfolgt 
durch Anpassung des Parameters $B$. Dieses wird durch folgende Überlegung verständlich: Fügt man dem Alterswert $\chi$ einen konstanten Zeitwert $\gamma$ hinzu, so lautet die Potenz von e : $\mathrm{e}^{-C(\gamma+\gamma)}=\mathrm{e}^{-C \chi} \cdot \mathrm{e}^{-C \gamma}$. Letztere Potenz von e stellt eine konstante Größe dar und tritt als Faktor zu dem Parameter $B$, der in dieser Weise die Anpassung an die Alterswerte übernimmt.

Der $\tau_{0}$-Wert der Bertalanffy-Funktion stellt den Zeitpunkt dar, zu dem rechnerisch der Organismus die Dimension Null hätte; diese mathematische Deutung war der Grund für seine Einführung. Biologisch ist sie aber nicht richtig, da kein Organismus aus dem Nichts entsteht, sondern schon in der Eizelle und davor als eine räumliche Struktur - z. B. in den Chromosomen - vorliegt. In dieser Beziehung entsprechen die relativen Wachstumsdarstellungen der Gompertz- und der Reziprok-Funktion, die keine Dimension Null zulassen, besser den biologischen Gegebenheiten.

$\zeta$ und $\tau_{0}$ stellen Grenzwerte dar, die für y Null beziehungsweise "unendlich klein" liefern und deren Unterschreitung keine biologisch sinnvolle Lösung ergibt.

Der $y_{0}$-Wert, dem wir sowohl in der Bertalanffy- als auch der GompertzFunktion begegnen, hat eine andere Bedeutung: er stellt nämlich die Dimension für den Wert 0 der angewandten Alterseinteilung dar, was der rechnerischen Größe bei der Geburt entsprechen würde. Man erkennt das leicht, wenn man für $\chi$ den Wert 0 einsetzt. Man erhält dann für die Bertalanffy-Funktion G1. (1):

$$
l_{0}=L_{\infty}-\left(L_{\infty}-L_{0}\right)
$$

und für die Gompertz-Funktion:

$$
\begin{array}{ll} 
& \ln y_{0}=\ln Y_{\infty}-B \\
\text { oder: } & B=\ln Y_{\infty}-\ln y_{0}
\end{array}
$$

Letztere Beziehung erscheint von besonderem Interesse, $\mathrm{da}$ sie in einfacher Weise die theoretische Dimension bei der Geburt zu berechnen gestattet, die durch Beobachtung verhältnismäßig leicht zu erfassen ist.

Weymouth \& McMillin geben die Länge der neugeborenen Muscheln mit 0,1 mm an. Ermittelt man aus den Gompertz-Parametern für die ganzen Meßreihen der Tabelle 2 die Werte für $y_{0}$, so erhält man die in Tabelle 3 (Spalte a) angegebenen Zahlen. Für die Muscheln aus Alaska entsprechen sie wenigstens in der Größenordnung den Erwartungswerten. Für die Muscheln aus den südlichen Gebieten ergeben sich dagegen - mit Ausnahme von "Copalis" - Werte, die erheblich unter 0,1 mm liegen, was besagt, daß die Altersangaben zu hoch angenommen wurden.

Man kann aber auch aus der Funktion errechnen, bei welchem Alter eine Länge von $0,1 \mathrm{~mm} \mathrm{zu}$ erwarten wäre und erhält auf diese Weise einen Hinweis auf die Fehlschätzung der eingegebenen Alterswerte. Mathematisch gegeben ist die Fehlschätzung $\left(\chi_{1}-\chi_{2}\right)$ mit:

$$
\chi_{1}-\chi_{2}=\frac{\ln B_{1}-\ln B_{2}}{C}
$$

( $\chi_{1}$ aus Parameter $B_{1}$ der Tabelle 2. $\chi_{3}$ und $B_{2}$ für die Geburtslänge von $0,1 \mathrm{~mm}$ korrigierte Werte).

Die erhaltenen Werte sind in Tabelle 3 (Spalte b) zusammengestellt. Für die Muscheln aus dem arktischen Gebiet ergaben sich aus den - Altersstufe 1 einschließen- 
Tabelle 3

Auf 0 bezogene - gekürzte - Werte der Gompertz- und der Reziprok-Funktion. Die Zahlen für $\gamma_{0}=0,01 \mathrm{~cm}$ sind für die Parameter in Spalte $\mathrm{c}$ und e von den Altersangaben der Tabellen von Weymouth et al. $(1930,1931)$ über das Wachstum von Siliqua patula abzuziehen beziehungsweise $\mathrm{zu}$ addieren

\begin{tabular}{|c|c|c|c|c|c|}
\hline \multirow[b]{2}{*}{ Populationen } & \multicolumn{3}{|c|}{ Gompertz-Funktion } & \multicolumn{2}{|c|}{ Reziprokfunktion } \\
\hline & $\begin{array}{c}y \text { für } z=0 \\
\text { cm } \\
\text { (a) }\end{array}$ & $\begin{array}{c}\text { Alter für } \\
\text { yo }=0,01 \mathrm{~cm} \\
\text { Alters- } \\
\text { korrektur } \\
\text { (b) }\end{array}$ & $\begin{array}{c}B \\
\text { korrigiert } \\
\text { (c) }\end{array}$ & $\begin{array}{c}\text { Alter für } \\
\text { yo }=0,01 \mathrm{~cm} \\
\text { Alters- } \\
\text { Korrektur } \\
\text { (d) }\end{array}$ & $\begin{array}{c}\chi_{0}-\xi \\
\text { (e) }\end{array}$ \\
\hline Pismo & 0,0007 & $-0,296$ & 7,122 & $-0,802$ & 0,082 \\
\hline Crescent & 0,0280 & $-0,129$ & 7,161 & $-0,678$ & 0,178 \\
\hline Channel & 0,0531 & $-0,072$ & 7,037 & $-0,619$ & 0,219 \\
\hline Sink & 0,00003 & $-0,399$ & 7,051 & $-0,840$ & 0,080 \\
\hline Copalis & 0,1352 & $+0,033$ & 7,208 & $-0,683$ & 0,123 \\
\hline Masset & 0,0143 & $-0,210$ & 7,164 & $-0,692$ & 0,232 \\
\hline Swickshak & 0,0822 & $-0,037$ & 7,304 & $-0,565$ & 0,495 \\
\hline Hallo Bay & 0,1071 & $+0,014$ & 7,299 & $-0,543$ & 0,553 \\
\hline Controller & 0,1626 & $+0,113$ & 7,064 & $-0,430$ & 0,670 \\
\hline Karl Bar & 0,1536 & $+0,095$ & 7,342 & $-0,499$ & 0,579 \\
\hline
\end{tabular}

den - Parametern in der Nähe von Null liegende beziehungsweise positive Werte. Die angenommenen Alterswerte wären demnach für sie zutreffend oder auch zu niedrig. Bei den südlichen Muschelpopulationen treffen wir mit Ausnahme von "Copalis" auf negative Fehlschätzungen. Die Altersangabe nach den Jahresringen wäre demnach zu hoch, allerdings nicht ganz so hoch, wie die Autoren annehmen. Man muß bei den Zahlen in Betracht ziehen, daß sie Extrapolationen über den ausgewerteten Bereich hinaus darstellen und daher nicht eindeutig und mit erheblicher Unsicherheit behaftet sind.

Die um die Fehlschätzung korrigierten Altersangaben erfordern natürlich für die Rückberechnung andere $B$-Werte, deren Beziehung zur Fehlschätzung durch G1. (14) gegeben ist. Im vorliegenden Fall, in dem die Geburtslänge $0,01 \mathrm{~cm}$ beträgt, liegen die Verhältnisse besonders einfach, denn es ist: $\ln Y_{\infty}-\ln 0,01=\ln 100 \cdot Y_{\infty}=B$. $\mathrm{Da}$ die Maximalwerte für alle Populationen sehr nahe beieinander liegen, betriff das auch die $B$-Werte, die für die südlichen Muscheln zwischen 7,04 und 7,21 und für die nördlichen Muscheln zwischen 7,06 und 7,34 schwanken (Tabelle 3, Spalte c).

$\mathrm{Zu}$ abweichenden Ergebnissen führt die Reziprokfunktion. Die in Tabelle 2 angegebenen $\xi$-Werte treffen nur für die Altersschätzung aus der Zahl der Zuwachsringe zu. Diese $\xi$-Werte setzen sich zusammen aus dem pränatalen Alter und der Korrektur für die Altersangabe, die im vorliegenden Fall dominiert. Die zumeist negativen Vorzeichen besagen, daß mathematisch gesehen die Entwicklung erst nach dem Alterswert $O$ begonnen hat. Auch hiernach sind die Altersangaben zu hoch anzusehen.

Für einen besseren Einblick müssen wir auch im vorliegenden Fall aus den Parametern der Tabelle 2 das rechnerische Alter für die Geburtslänge von 0,1 mm errech- 
nen. Die erhaltenen Zahlen sind in Tabelle 3 (Spalte d) aufgenommen und geben an, um welche Werte die Altersstufen zu hoch angenommen wurden. Den für die korrigierten Alterswerte zutreffenden $\zeta$-Wert erhält man bei negativem Vorzeichen durch Subtraktion des Ausgangswertes von dem Alter für $y=0,01 \mathrm{~cm}$. Er soll als "korrigierter" $\xi$-Wert bezeichnet werden. Er ist sinnvollerweise in allen Fällen positiv, für die arktischen Populationen höher als für die südlichen.

Die Alterskorrekturen, die im Mittel etwa 0,7 betragen und um den die ursprünglichen Alterswerte zu vermindern sind, erscheinen für die südlichen Muscheln recht hoch. Für die nördlichen Muscheln liegen sie im Bereich der Schätzung der Autoren.

Die korrigierten $\zeta$-Werte differieren nicht sehr erheblich und liegen in ihrer Größenordnung durchaus im Bereich einer pränatalen Entwicklungszeit. Ein sehr deutlicher Unterschied tritt in den Werten für die nördlichen und südlichen Populationen zutage.

Unabhängig von der Deutung von $\zeta$ als pränatales Alter besitzt dieser Parameter noch einen rein mathematischen Aussagewert, nämlich als Krümmungsparameter für die relativen Wachstumskurven. Niedere $\zeta$-Werte beschreiben stark gekrümmte Kurven mit steilem anfänglichen Kurvenanstieg, der sich sehr bald abflacht und schon früh dem Endwert nähert. Die reduzierten $\zeta$-Werte liegen recht niedrig und sprechen für ein sehr schnelles Anfangswachstum, das aber bei den arktischen Beständen etwas langsamer abläuft.

An dieser Stelle erhebt sich die Frage, in welchem Parameter bei der GompertzFunktion dieser Unterschied im Kurvenverlauf zum Ausdruck kommt. $\mathrm{Zu}$ ihrer Klärung habe ich die reduzierten $\zeta$-Werte der Tabelle 3 der Höhe nach geordnet und ihnen die entsprechenden C-Werte der Gompertz-Funktion gegenübergestellt. Es ergab sich eine volle Ubereinstimmung in der Rangordnung, allerdings mit dem Unterschied, daß bei der Gompertz-Funktion die höheren Werte stärkeren Kurvenkrümmungen entsprechen und umgekehrt. Zu den B-Werten ergab sich keine entsprechende Korrelation, so daß dem Parameter $\mathrm{C}$ alleine die Rolle als Krümmungsparameter zuzuschreiben ist.

Bei der Bertalanffy-Funktion entspricht die Folge der K-Werte der Ordnung der $\zeta$ - und C-Werte, so daß man bei ihr in diesem Parameter den Ausdruck für die Kurvenkrïmmung sehen muß. Durch das Fehlen des Wendepunktes fällt bei der Bertalanffy-Funktion der Anfangsteil der Kurve so steil ab, daß sich keine sinnvollen Zeitangaben für die Geburt ergeben.

\section{DER WENDEPUNKT}

Es ist seit langem bekannt, daß in typischen Fällen die graphische Auftragung linearer Wachstumsdaten als Funktionen der linearen Alterswerte zu Sigmoid-Kurven führt. Bei dem vorliegenden Datenmaterial zeigen die Muscheln aus Alaska einen solchen Verlauf, aber nicht immer ist in den Wachstumsdaten der Wendepunkt deutlich ausgeprägt. Es triff dieses vor allem bei Daten für das Längenwachstum zu. 
Die ungefähre Lage des Wendepunktes läßt sich aus den Daten ablesen, wenn man die linearen Differenzen zwischen den einzelnen Werten als Funktion des Alters aufträgt und nach Augenmaß eine Kurve durch die erhaltenen Punkte zieht. Die auf diese Weise erhaltenen Schätzwerte sind in Tabelle 2 wiedergegeben. Bei den Muscheln südlicher Herkunft liegt der Wendepunkt in der Nähe von 1,5 Jahren für die Altersangaben der Tabellen. Bei den arktischen Muscheln liegt er dagegen bei einem Alter von etwa 3 Jahren.

Die semilogarithmische Darstellung der Logarithmen der Dimensionen als Funktion der linearen Alterswerte liefert eine Kurve, die im Anfangsteil steil ansteigt, mit zunehmendem Alter sich immer mehr abflacht und keinen Wendepunkt aufweist. Sie nähert sich asymptotisch einem Maximalwert. Diese "relative" Wachstumskurve stellt gleiche prozentuale Zuwachsraten durch gleiche Steigungen dar. Ihre Abflachung besagt also, daß im Verlauf des Wachstums die auf die jeweilige Größe bezogene relative (prozentuale) Geschwindigkeit sich kontinuierlich vermindert und dem Wert Null nähert. Weymouth et al. $(1930,1931)$ haben klar erkannt, daß bei der Auftragung der linearen Werte dieser Kurve ein Wendepunkt auftritt.

Der Wendepunkt stellt also eine rein mathematische Konsequenz der im Verlauf des Wachstums abnehmenden relativen Wachstumsraten dar, und es bleibt unverständlich, daß bis in die neueste Zeit hinein (Scharf, 1969) diese Erkenntnis unbeachtet blieb und der Wendepunkt als Ausdruck besonderer Umstellungen im Organismus angesehen wird.

Scharf (1969) benutzte für die Darstellung des Wendepunktes beim Längenwachstum des menschlichen Embryo eine Funktion, die den Tangens hyperbolicus enthält. Hoeppe (1959) wendet gegen dieses Vorgehen, das zuvor schon Smith (1954) vorschlug, mit Recht ein, daß der Tangens eine um den Wendepunkt symmetrische Kurve beschreibt, eine solche Symmetrie aber für Wachstumskurven nicht zutriff.

Die genaue Lage des Wendepunktes ergibt sich für Kurven, denen eine bekannte Funktion zugrunde liegt, aus dem zweiten Differentialquotienten. Bei der GompertzFunktion erhält man hierfür:

$$
\chi \text { Wendepunkt }=\frac{\ln B}{C}
$$

Für die Reziprokfunktion ergibt sich:

$$
\chi \text { Wendepunkt }=\frac{\ln N}{2}-\zeta
$$

Ein Vergleich des aus der Berechnung sich ergebenden Zeitpunktes mit dem in den Meßwerten auftretenden Wendepunkt kann als weiteres Kriterium für die Beurteilung einer Wachstumsfunktion dienen. In Tabelle 2 sind daher für die Gompertzund die Reziprok-Funktion die aus den ermittelten Parametern erhaltenen Zahlen für die zeitliche Lage des Wendepunktes wiedergegeben. Die Parameterberechnung erfolgt bei beiden Funktionen übereinstimmend auf der Basis der relativen Wachstumskurven.

Wie ein Vergleich mit der in Tabelle 2 wiedergegebenen geschätzten Lage der Wendepunkte erkennen läßt, kommen die Werte der Gompertz-Funktion den wirklichen Verhältnissen durchweg sehr nahe. Demgegenüber ergeben sich aus der Rezi- 
prokfunktion in allen Fällen zu niedrige Werte. Hinsichtlich der Lage des Wendepunktes bietet also die Reziprokfunktion - trotz ihrer zumeist besseren Näherung an gegebene Wachstumsdaten - keine befriedigende Lösung.

\section{DIE WACHSTUMSGESCHWINDIGKEIT}

Die Geschwindigkeit, mit der Organismen wachsen, stellt eine Größe dar, deren zahlenmäßige Erfassung für die Bearbeitung zahlreicher Fragen von Bedeutung wäre. Der Weg hierzu kann nur über mathematische Wachstumsbeschreibungen führen. Statistische Verfahren erscheinen bei der Inkonstanz der Wachstumsraten nur begrenzt anwendbar. Wir müssen allerdings feststellen, daß mit den Modellen alleine das Problem noch nicht gelöst ist. Keine der Funktionen enthält einen Parameter, der eine eindeutige Aussage über die Wachstumsgeschwindigkeit gestattet. Auch die als Wachstumskonstanten bezeichneten Parameter erfüllen diese Aufgabe nicht.

Die Ursache liegt darin, daß es keine einheitliche Wachstumsgeschwindigkeit gibt, sondern daß sie sich ständig - absolut und relativ - ändert. Durch die Differentialquotienten der Funktionen [Gl. (22) und Gl. (24)] läßst sich zwar die Wachstumsgeschwindigkeit ermitteln, aber nur für bestimmte Zeitpunkte, und die Berechnung erfordert neben der Angabe des Alters den Einsatz praktisch aller Parameter.

Es war schon Weymouth et al. (1930) aufgefallen, daß die aus dem Süden stammenden Muscheln von "Pismo" anfänglich sehr viel schneller wachsen als ihre Artgenossen aus Alaska. Sie sind im zweiten Jahr schon so groß wie die Muscheln aus der Arktis erst mit 4 Jahren. Nach dieser Zeit aber übertreffen die Alaska-Muscheln das Wachstum der südlichen Bestände und werden im Alter zumeist auch größer.

Die Beobachtung verdeutlicht an einem konkreten Fall die Schwierigkeit einer eindeutigen Aussage über die Wachstumsgeschwindigkeit, sie läßt sich aber anhand der Wachstumsmodelle leicht verständlich machen. Die Dimension am Wendepunkt steht bei der Gompertz- und der Reziprok-Funktion in einem festen Verhältnis zur Maximaldimension und hängt ausschließlich von ihr ab. Bei der Gompertz-Funktion ist die Größe beim Wendepunkt gegeben durch:

$$
y_{\text {Wendepunkt }}=\frac{A}{\mathrm{e}}=\mathrm{ca} .0 .368 Y_{\infty}
$$

Für die Reziprokfunktion beträgt die Größe beim Wendepunkt:

$$
y_{\text {Wendepunkt }}=\frac{Y_{\max }}{\mathrm{e}^{2}}=c a \cdot 0,135 Y_{\infty}
$$

Bei früher Lage des Wendepunktes, wie er für die südlichen Muscheln zutriff, wird diese Größe schneller erreicht, also ist ihre Wachstumsgeschwindigkeit höher. Das Wachstum ist dann aber infolge der Begrenzung durch den angestrebten Maximalwert verlangsamt. Bei einem später liegenden Wendepunkt finden wir dementsprechend ein verlangsamtes Wachstum vor und ein beschleunigtes Wachstum nach dem Wendepunkt. Die Befunde an den verschiedenen Populationen von Siliqua patula bestätigen die mathematischen Erwägungen, wobei allerdings zusätzlich der Unterschied noch 
deutlicher gemacht wird durch die geringeren Maximalgrößen der Muscheln südlicher Herkunft.

Für die Bertalanffy-Funktion hat Hohendorf (1966) abgeleitet, daß der Parameter $K$ einen dimensionslosen Zahlenwert darstellt, der von der Wachstumsgeschwindigkeit abhängt und daher als "Wachstumskonstante" bezeichnet werden kann. Das gleiche triff auch auf den Parameter $C$ der Gompertz- und den Parameter $N$ der Reziprok-Funktion zu. Aber alle drei Geschwindigkeitskonstanten stellen für sich alleine keine exakten und vergleichbaren Zahlenwerte dar, sondern nur in Kombination mit anderen Parametern. Sie sind, wie oben besprochen wurde, unabhängig von Fehlschätzungen des Alters und können daher direkt der Tabelle 2 entnommen werden. Aus ihr kann man erkennen, daß die Parameter $K$ und $C$ eine allerdings nicht ganz strenge Tendenz zur Abnahme vom Süden zum Norden erkennen lassen. Die Werte für $N$ - und damit auch für $\log N$ - steigen dagegen nach Norden hin; sie liegen für die südlichen Bestände unter 1,0 und für die Muscheln aus Alaska über 1,0.

Die Frage, wieweit diese Unterschiede lediglich auf den unterschiedlichen Temperaturbedingungen beruhen, oder ob auch genetische Unterschiede durch Rassenbildung vorliegen, läßt sich auf diesem Wege naturgemäß nicht beantworten.

Für eine genauere Erfassung der Wachstumsgeschwindigkeiten, wie sie wohl schon Weymouth und seine Mitarbeiter ins Auge gefaßt hatten, sind die Wachstumskonstanten offensichtlich ungeeignet. Hier müssen wir nach anderen Möglichkeiten suchen.

Die nächstliegende Lösung stellt die Halbwertzeit dar, also die Zeit, die benötigt wird, um auf den halben Wert der mathematischen Maximalgröße heranzuwachsen. Sie umfaßt das ganze Wachstum vor dem Wendepunkt und einen Teil der Wachstumsgeschwindigkeit nach dem Wendepunkt. Höhere Werte kennzeichnen verständlicherweise geringere Geschwindigkeiten und umgekehrt. Aus den Parametern der Gompertz-Funktion errechnet sie sich zu:

$$
\text { Halbwertzeit }=\frac{\ln B-\ln \ln 2}{C}
$$

Für die Pütter-Bertalanffy-Funktion ergibt sie sich zu:

$$
\text { Halbwertzeit }=\frac{\ln 2}{K}+\tau_{0}
$$

Für die Reziprokfunktion erhält man:

$$
\text { Halbwertzeit }=\frac{\log N}{\log 2}-\zeta
$$

Die in Tabelle 4 wiedergegebenen Halbwertzeiten liefern für die Gompertzund die Bertalanffy-Funktion, deren Maximalwerte nicht sehr unterschieden sind, dicht beieinander liegende Zahlen. Entsprechend den höheren Maximalwerten der Reziprokfunktion liegen - mit einer Ausnahme - die aus ihren Parametern errechneten Halbwertzeiten höher. Hinsichtlich der relativen Bewertung der Wachstumsgeschwindigkeiten stimmen die Halbwertzeiten der drei Funktionen für die verschiedenen Fundorte vollkommen überein. Eine besonders hohe Wachstumsgeschwindigkeit findet sich neben den Muscheln von "Pismo" bei den Muscheln von "Sink" und "Copalis". Die niedrigsten Wachstumsgeschwindigkeiten weisen die Muscheln aus 
Alaska und hier besonders von "Controller" auf. Für einen allgemeiner gültigen Vergleich sind die Halbwertzeiten ungeeignet, da sie zu stark von der Endgröße abhängen.

Wie oben betont wurde, macht es die fortlaufende Änderung der Wachstumsgeschwindigkeit unmöglich, sie für die ganze Wachstumsperiode durch eine einzelne Zahl zu kennzeichnen. Sie läßt sich nur für einzelne Zeitpunkte durch den Differentialquotienten festlegen. Als solcher erscheint der Wendepunkt der Wachstumskurve bevorzugt, an dem die Wachstumsgeschwindigkeit ihr Maximum erreicht. Es ist günstig, daß sich hierfür sehr einfache Lösungen ergeben.

Der Differentialquotient der Gompertz-Funktion lautet:

$$
\frac{\mathrm{d} y}{\mathrm{~d} \chi}=y \cdot B \cdot C \cdot \mathrm{e}^{-\mathrm{C} \cdot \chi}
$$

Setzt man in ihn die für y und $\chi$ aus Gl. (15) und Gl. (17) sich ergebenden Lösungen ein, so erhält man für die Maximalgeschwindigkeit im Wendepunkt:

$$
\text { Maximalgeschwindigkeit }=\frac{A \cdot C}{\mathrm{e}}
$$

Für den Differentialquotienten der Reziprokfunktion:

$$
\frac{\mathrm{d} y}{\mathrm{~d} \chi}=y \cdot \frac{\ln N}{(\chi+\zeta)^{2}}
$$

erhält man in entsprechender Weise, wenn man Gl. (16) und Gl. (18) einsetzt:

$$
\text { Maximalgeschwindigkeit }=\frac{4 \cdot Y_{\max }}{\mathrm{e}^{2} \cdot \ln N}=0,2351 \frac{Y_{\max }}{\log N}
$$

Bei beiden Funktionen beschränkt sich die Beschreibung der Maximalgeschwindigkeit am Wendepunkt auf zwei Parameter. Bei der Reziprokfunktion ist besonders bedeutungsvoll, daß der additive Alterswert $\zeta$ ausgeschaltet ist und daher auch ein Vergleich unterschiedlicher Kurvenformen möglich erscheint. Wie die Verhältnisse bei der Gompertz-Funktion liegen, ist nicht klar, da bei ihr der Parameter $\mathrm{C}$ als Krümmungsparameter fungiert.

Die aus den Parametern der Tabelle 2 errechneten Maximalgeschwindigkeiten sind zur Erleichterung eines Vergleichs neben den Halbwertzeiten in Tabelle 4 wiedergegeben. Höhere Zahlen kennzeichnen höhere Wachstumsgeschwindigkeiten und umgekehrt.

Die Anordnung der Fundorte erfolgte in Tabelle 4 nach der Höhe der errechneten Maximalgeschwindigkeiten. Sie läuft bei der Gompertz- und der ReziprokFunktion genau parallel und mit Ausnahme von "Channel" und "Masset" auch parallel den Halbwertzeiten. Die - wie die Tabelle zeigt - mehrfach bestätigten Aussagen über das relative Verhältnis der Wachstumsgeschwindigkeiten dürtte demnach zutreffend sein und geeignet als Grundlage für eine Diskussion der Ergebnisse. zu dienen.

Ganz eindeutig ergibt sich die höchste Wachstumsgeschwindigkeit für die Muscheln des südlichsten Fundortes bei „Pismo“. Ursache dürfte wohl die hohe durchschnittliche Wassertemperatur in dieser Breite sein. Sie ist aber nicht alleine bestimmend für die Wachstumsgeschwindigkeit. Die Fundorte "Sink" und "Channel" lie- 
Tabelle 4

Vergleich der Zahlen der berïcksichtigten Funktionen für die Halbwertzeiten und die Maximalgeschwindigkeiten am Wendepunkt für das Wachstum von Siliqua patula

\begin{tabular}{|lccccc|}
\hline Populationen & $\begin{array}{c}\text { Gompertz- } \\
\text { funktion }\end{array}$ & $\begin{array}{c}\text { Halbwertzeiten } \\
\text { Bertalanffy- } \\
\text { funktion }\end{array}$ & $\begin{array}{c}\text { Reziprok- } \\
\text { funktion }\end{array}$ & $\begin{array}{c}\text { Maximalgeschwindigkeiten } \\
\text { Gompertz- } \\
\text { funktion }\end{array}$ & $\begin{array}{c}\text { Reziprok- } \\
\text { funktion }\end{array}$ \\
\hline Pismo & 1,57 & 1,49 & 1,58 & 8,33 & 13,12 \\
Sink & 1,62 & 1,53 & 1,59 & 8,06 & 12,58 \\
Copalis & 1,77 & 1,72 & 1,86 & 6,46 & 9,53 \\
Crescent & 1,97 & 1,98 & 2,41 & 6,00 & 6,88 \\
Massett & 2,26 & 2,37 & 2,96 & 5,43 & 5,40 \\
Channel & 2,03 & 2,11 & 2,72 & 4,96 & 5,38 \\
Schwickshak & 3,46 & 3,50 & 4,25 & 3,45 & 4,44 \\
Hallo Bay & 3,68 & 3,73 & 4,58 & 3,28 & 3,99 \\
Karl Bar & 3,81 & 3,91 & 5,24 & 3,18 & 3,58 \\
Controller & 3,96 & 4,68 & 6,58 & 2,25 & 2,38 \\
\hline
\end{tabular}

gen dicht benachbart bei "Copalis", so daß die Temperaturbedingungen praktisch identisch sein dürften, sie unterscheiden sich aber stärkstens hinsichtlich der Wachstumsgeschwindigkeit. Die Wachstumsgeschwindigkeit bei "Sink" ist die höchste nach "Pismo". Der Standort ist nach Weymouth \& McMillin (1930) gekennzeichnet durch einen stark schlickhaltigen Sand und fehlende Brandung. In schärfstem Gegensatz hierzu steht das sehr langsame Wachstum der Muscheln von "Channel", deren Standort kiesig und starken Strömungen ausgesetzt ist. Wenn die Autoren den von der Brandung ausgewaschenen Sand von "Copalis" für besonders günstig halten, so triff das hinsichtlich der Wachstumsgeschwindigkeit nicht ganz zu. Immerhin aber übertriff sie die Wachstumsgeschwindigkeit der weiter südlich bei "Crescent" gefundenen Bestände, die vermutlich bei höheren Wassertemperaturen leben. Die geringe Wachstumsgeschwindigkeit der Bestände von "Masset" dürfte auf die nördliche Lage des Fundortes zurückzuführen sein. Das triff natürlich in noch höherem Maße auf die Muscheln aus Alaska zu, für die die Autoren aber keine hinreichende Beschreibung der Fundorte geben.

Die mathematische Analyse läßt also als Faktoren, die das Wachstum der Muscheln beeinflussen, die Temperatur und Umweltbedingungen und wahrscheinlich das Nahrungsangebot erkennen. Die Überlagerung dieser Faktoren, die vermutlich in unterschiedlicher Weise das Wachstum beeinflussen, macht den sehr verschieden gestalteten Verlauf der Wachstumskurven von den verschiedenen Fundorten verständlich.

\section{DIE ALLOMETRIEBEZIEHUNG}

Die Aufdeckung der Oberflächenabhängigkeit des Stoffwechsels durch Rameaux \& Sarrus (1837/39, zitiert nach Thompson, 1952) brachte erstmals die Erkenntnis, daß tierischen Wachstumsprozessen - mathematisch gesehen - Exponentialfunktionen 
zugrunde liegen. Sie gipfelte in der Aufstellung der heute als allometrische Funktion bezeichneten Formel durch Snell (1891) und Dubois (1897).

Nachdem sich die von Huxley \& Teissier (1936) vorgeschlagene Schreibung der Funktion nicht durchsetzen konnte, benutze ich sie in neuerer Zeit in der Form:

$$
y=a \cdot x^{\beta}
$$

In ihr stellen $x$ und $y$ die verglichenen Dimensionen dar; a ergibt sich für $x=1$; $\beta$ stellt den allometrischen Exponenten dar, der die quantitive Beziehung zwischen den verglichenen Dimensionen wiedergibt. Durch Logorithmierung geht die Funktion in die Gleichung einer Geraden über, die zur Ermittlung der beiden Parameter a. und $\beta$ dient.

Die allometrische Funktion beschreibt die Veränderung der relativen Größe zweier Dimensionen eines wachsenden Organismus unter Ausschaltung des Alters und stellt eine allgemeine Formulierung für die Beziehung zwischen den Dimensionen geometrisch ähnlicher Körper dar.

Biologisch besitzt großes Interesse das Verhältnis der Lineardimension zum Volumen oder mit anderen Worten: das Verhältnis von Länge zu Gewicht. Theoretisch wäre in diesem Fall für den Exponenten $\beta$ der Wert 3,0 zu erwarten, wenn das Wachstum ohne Veränderungen der Gestalt abläuft. Diese Bedingung scheint in vielen Fällen auch erfüllt zu sein. Weymouth und seine Mitarbeiter haben ihre Messungen auf die Bestimmung einer Lineardimension beschränkt, so daß das Gewicht der Muscheln unbekannt ist.

Da mir aber die Aufklärung der Beziehung der Gompertz-Funktion zux allometrischen Funktion sehr bedeutungsvoll erscheint, möchte ich diese Frage an dem Zahlenmaterial behandeln, das Sella (1929) über das Wachstum des Thunfisches veröffentlichte. Es umfaßt das Längen- und Gewichts-Wachstum über 14 Altersstufen, also eine recht weite Spanne, die für die Testung von Wachstumsfunktionen geeignet erscheint. Die allometrische Auswertung liefert für den Exponenten $\beta$ mit 2,9973 fast den theoretischen Wert; für $a$ ermittelt sich in Gramm ausgedrückt der Wert 0,0167.

$\mathrm{Da}$ die von mir angegebenen Gompertz-Parameter für das Wachstum des Thunfisches durch Variation des Maximalwertes ermittelt wurden (Krüger, 1973), ein Verfahren, dem gewisse Bedenken gegenüberstehen, habe ich sie entsprechend der im methodischen Abschnitt geschilderten Weise neu berechnet. Das neue Verfahren ergab eine etwas geringere Standardabweichung bei nicht sehr erheblich veränderten Parameterwerten, über die Tabelle 5 eine Übersicht gibt.

Die Beziehung von Wachstumsfunktionen zur allometrischen Funktion setzt voraus, daß die zu vergleichenden Dimensionen als Funktion eines identischen Alterswertes beschrieben werden können, um diesen zu eliminieren. Formal ergibt sich hierbei folgende Beziehung zwischen Gompertz- und Allometrie-Parametern:

$$
\ln a=\ln W_{\infty}-\beta \cdot \ln L_{\infty} ; \quad \beta=\frac{B_{\mathrm{w}}}{B_{1}}
$$

Um die Bedingung eines identischen Alterswertes zu erfüllen, habe ich die Zahlenreihen für Länge und Gewicht auf der Basis eines gemeinsamen mittleren Wertes von $C=0,1592$ ausgewertet. Die Zahlen sind in Tabelle 5 wiedergegeben. Fïr beide Dimensionen ergab sich durch den vom Optimum abweichenden $C$-Wert keine 
Tabelle 5

Parameter für das Längen- und Gewichtswachstum nach den Daten von Sella für das Wachstum von Thunnus thynnus

\begin{tabular}{|c|c|c|c|c|}
\hline \multicolumn{2}{|l|}{ Allometrische Funktion } & \multirow{2}{*}{$\frac{a}{0,01675 \mathrm{~g}}$} & \multirow{2}{*}{$\frac{\beta}{2,9973}$} & \multirow{2}{*}{$\frac{s \%}{4,56}$} \\
\hline & & & & \\
\hline Gompertz-Funktion & $A$ & $B$ & $C$ & $5 \%$ \\
\hline Länge (optimal) & 292,696 & 1,7916 & 0,1670 & 1,47 \\
\hline Länge (interpoliert) & 301,205 & 1,7999 & 0,1592 & 1,57 \\
\hline Gewicht (optimal) & 496,194 & 5,4331 & 0,1514 & 4,63 \\
\hline Gewicht (interpoliert) & 451,042 & 5,3978 & 0,1592 & 4,95 \\
\hline \multicolumn{5}{|c|}{$\beta=\frac{B_{\mathrm{W}}}{B_{1}}$ (interpolierte Werte) $=2,9989$} \\
\hline Reziprokfunktion & $Y_{\max }$ & $\log N$ & $\zeta$ & $s \%$ \\
\hline Länge (optimal) & 547,863 & 6,9343 & 6,40 & 1,36 \\
\hline Länge (interpoliert) & 586,454 & 7,6895 & 7,00 & 1,51 \\
\hline Gewicht (optimal) & 3761,874 & 24,4727 & 7,36 & 3,20 \\
\hline Gewicht (interpoliert) & 3330,323 & 23,0663 & 7,00 & 3,38 \\
\hline$\beta=\frac{\log N_{\mathrm{w}}}{\log N_{\mathrm{l}}}$ (in & e Werte) & 997 & & \\
\hline
\end{tabular}

sehr erhebliche Verschlechterung der prozentualen Abweichung. Das Verhältnis $B_{\mathrm{W}} / B_{1}=2,999$ lieferte fast den gleichen Wert wie die Allometrie-Auswertung. Hiermit ist die Ableitung der allometrischen Beziehung aus der Gompertz-Funktion auch numerisch bestätigt.

In Tabelle 5 sind zum Vergleich die für die Reziprokfunktion einzusetzenden Parameterwerte aufgenommen. Hinsichtlich der angegebenen prozentualen Standardabweichung zeigt sie sich auch gegenüber den neu ermittelten Gompertz-Parametern etwas überlegen. Wir müssen aber wieder feststellen, daß sie die Lage des Wendepunktes für das Längenwachstum unzutreffend wiedergibt. Die höchste Zuwachsrate liegt zwischen den Altersstufen 3 und 4. Aus den Parametern der Reziprokfunktion errechnet sich seine Lage bei 1,57 Jahren, also wesentlich zu niedrig. Demgegenüber liefert die Gompertz-Funktion mit 3,49 Jahren einen wesentlich besseren Wert.

Man darf aber die bessere Schätzung der Lage des Wendepunktes durch die Gompertz-Funktion nicht verallgemeinern. Die Daten für das Gewichtswachstum zeigen bis zur höchsten Altersstufe von 14 Jahren steigende Zuwachsraten, so daß also der Wendepunkt später liegen muß. Wenn in diesem Fall sich seine Lage aus der Gompertz-Funktion zu 11,18 Jahren errechnet, so ist das also sicher unzutreffend. Ob der Wert von 20,78 Jahren, den die Reziprokfunktion angibt, richtig ist, entzieht sich leider einer Nachprüfung.

An dieser Stelle sei noch eine Bemerkung eingeflochten. Aus den Parametern aller drei benutzten Funktionen errechnet sich für den Alterswert 0 - also die Länge bei der Geburt - eine Länge von etwa $45 \mathrm{~cm}$. Das ist natürlich ein absolut unmög- 
licher Wert und es liegt daher die Vermutung nahe, daß die Altersschätzungen in den Tabellen nicht zutreffend sind. Hieraus würde auch der außerordentlich hohe Wert verständlich, der sich bei der Reziprokfunktion für das pränatale Alter ergab.

\section{ALLOMETRIE-SUMMATION}

Mit der Ableitung der allometrischen Beziehung aus der Gompertz-Funktion erschien es erforderlich, zu prüfen, ob sie auch eine Lösung für das Problem der Allometrie-Summation zuläßt. Hierbei handelt es sich um die Frage, ob sich mehrere allometrische Funktionen zu einem Ganzen summieren lassen, das wieder durch eine allometrische Funktion wiedergegeben werden kann. Es ist dieses ein Problem, das lange Zeit diskutiert und für unlösbar gehalten wurde. Ausgehend von der Reziprokfunktion gelang es mir (Krüger, 1970), hierfür eine Lösung zu geben und ich gewann den Eindruck, daß man sie auch ausgehend von der Gompertz-Funktion erhalten könnte.

Das Vorgehen entspricht dem bei der Reziprokfunktion angewandten Verfahren. Stellen wir die aus Teilen zusammengesetzte Größe y:

$$
y_{1}+y_{2}+y_{3}+\cdots y_{\mathrm{n}}=y
$$

mit Hilfe der Gompertz-Funktion als Funktion des Alters dar, so erhalten wir:

$$
A_{1} \mathrm{e}^{-B_{1} \mathrm{e}^{-C_{\chi}}}+A_{2} \mathrm{e}^{-B_{2} \mathrm{e}^{-C_{\chi}}}+A_{3} \mathrm{e}^{-B_{3} \mathrm{e}^{-C_{\chi}}}+\cdots A_{\mathrm{n}} \mathrm{e}^{-B_{\mathrm{n}} \mathrm{e}^{-C_{\chi}}}=A \mathrm{e}^{-B^{-} \mathrm{e}^{-C_{\chi}}}
$$

Differenzieren wir diese Summe, ergibt sich:

$$
\frac{\mathrm{d} y}{\mathrm{~d} \chi}=y_{1} B_{1} C \mathrm{e}^{-C_{\chi}}+y_{2} B_{2} C \mathrm{e}^{-C_{\chi}}+y_{3} B_{3} C \mathrm{e}^{-C_{\chi}}+\cdots \cdot y_{\mathrm{n}} B_{\mathrm{n}} C \mathrm{e}^{-C_{\chi}}=y B C \mathrm{e}^{-C_{\chi}}
$$

Wie wir sahen, ist für das Zutreffen der allometrischen Beziehung Voraussetzung, daß die verglichenen Wachstumsprozesse als Funktion eines identischen Alterswertes dargestellt werden können. Im vorliegenden Falle besagt dieses, das neben dem Alterswert $\chi$ auch der Parameter $C$ für alle einzelnen Glieder übereinstimmt. Durch Division mit $B C \mathrm{e}^{-C_{\chi}}$ geht Gl. (30) dann über in:

$$
y_{1} \frac{B_{1}}{B}+y_{2} \frac{B_{2}}{B}+y_{3} \frac{B_{3}}{B}+\cdots y_{\mathrm{n}} \frac{B_{\mathrm{n}}}{B}=y
$$

Nun wissen wir, daß der Quotient $\frac{B_{1}}{B_{2}}$ den allometrischen Exponenten $\beta$ darstellt. Außerdem sind $y_{1}, y_{2}$ usw. Dimensionen einer beliebigen Altersstufe. Daher können wir an ihrer Stelle auch die a-Werte einsetzen und erhalten dann die Gleichung:

$$
a_{1} \beta_{1}+a_{2} \beta_{2}+a_{3} \beta_{3}+\cdots a_{n} \beta_{n}=1
$$

Die 1 für die Summe der Produkte der allometrischen Parameter ergibt sich dadurch, daß die a-Werte auf die Einheit der Vergleichs-Dimension bezogen werden.

Die erhaltene Lösung der Allometrie-Summation ist identisch mit der, die ich ausgehend von der Reziprokfunktion erhalten hatte (Krüger, 1970). Beim Vergleich der beiden Arbeiten muß man aber beachten, daß ich in der Zwischenzeit die Sym- 
bole für die beiden Parameter der allometrischen Funktion geändert habe. In der damaligen Arbeit hatte ich auch an einem konkreten Beispiel das Zutreffen der mathematischen Ableitung bestätigen können und außerdem, daß sich für die Summe der $a$-Werte 1 ergibt:

$$
a_{1}+a_{2}+a_{3}+\cdots a_{\mathrm{n}}=1
$$

Die aus zwei unterschiedlichen Funktionen erhaltene identische Lösung macht wohl sicher, daß in Gl. (32) eine Grundregel für die Entwicklung der Körperproportionen eines wachsenden Organismus gegeben ist, auch wenn sie - wie in der damaligen Arbeit gezeigt wurde - nur eine sehr gute Näherung darstellt.

\section{DISKUSSION DER AUSWERTUNGEN}

Die Rasiermesser-Muschel (razor clam) dient in Nord-Amerika als Speisemuschel. Daher war man an ihrem Wachstumsverhalten interessiert, und Weymouth und seine Mitarbeiter konnten ihre Messungen an einem sehr umfangreichen Material von $10 \mathrm{klimatisch} \mathrm{sehr} \mathrm{unterschiedlichen} \mathrm{Fundplätzen} \mathrm{durchführen.} \mathrm{Ihre} \mathrm{Meßwerte}$ darf man also in den biologisch gegebenen Grenzen als zuverlässig ansehen.

Ihr Zahlenmaterial weist allerdings den Mangel auf, daß es nur Längenmessungen bietet. Das Grundphänomen jeden Wachstums bildet aber die Zunahme der lebendigen Masse, der gegenüber die Vergrößerung der Lineardimensionen eine selsundäre Erscheinung darstellt. Ein Rückschluß auf das Massenwachstum wäre nur möglich bei Kenntnis der quantitativen Beziehungen zwischen Lineardimension und Gewicht. Die erheblichen Anderungen der Schalenproportionen im Verlauf des Wachstums sprechen gegen einfachere exponentielle Beziehungen, wie sie durch die allometrische Funktion erfaßt werden können. Hierdurch lassen sich vielleicht einige Schwierigkeiten erklären, die sich bei der Auswertung ergaben. Vermutlich stellen also die Daten für das nicht-isometrische Wachstum von Siliqua patula keine "normal“ verlaufenden Wachstumskurven dar.

Es liegt eine unübersehbare Zahl von Vorschlägen für Wachstumsformeln vor, unter denen die Pütter-Bertalanffy-Funktion allgemeinere Anerkennung gefunden hat. Daher enthalten auch zahlreiche Wachstumsuntersuchungen Angaben über die Parameter dieser Funktion. Demgegenüber wurde die Gompertz-Funktion nur selten Wachstumsbeschreibungen zugrunde gelegt. Schwierigkeiten bei der mathematischen Auswertung dürften hierfür verantwortlich gewesen sein. Die ansehnliche Zahl von Veröffentlichungen, die sich mit der Parameter-Bestimmung der Wachstumsfunktionen befassen, lassen die bis in die neueste Zeit hineinreichenden Schwierigkeiten erkennen.

Die vorliegende Untersuchung bestätigte, daß auch die Gompertz-Funktion zur Approximation von Wachstumskurven geeignet ist. Im Grunde wäre sie der Bertalanffy-Funktion vorzuziehen, da sie sich auch zur Wiedergabe von Kurven mit einem Wendepunkt eignet.

Bei den Daten für das Wachstum der Muscheln aus der Arktis mit einem ziemlich spät gelegenen Wendepunkt erwies sich die Gompertz-Funktion deutlich als überlegen. Die Frage, in welchem Umfang das nicht-isometrische Wachstum diesen Vor- 
rang begünstigt, muß offen bleiben. Für die Darstellung der Kurven mit sehr frühem Wendepunkt war sie weniger gut geeignet.

Eine eindeutig bevorzugte Stellung kann man keiner der drei berücksichtigten Funktionen zuschreiben. Die Pütter-Bertalanffy-Funktion lieferte zwar zumeist sehr gute Näherungen, man hat aber doch das Fehlen eines Wendepunktes als einen grundsätzlichen Mangel anzusehen. Mit Hoeppe (1959) muß man fordern, daß ein wirklich brauchbares Wachstums-Modell den Wendepunkt einschließt, der in zahlreichen Wachstumskurven auftritt. Die Gompertz-Funktion erfüllt zwar diese Bedingung, jedoch erscheint bei ihr der Abstand zwischen den höchsten Meßwerten und dem oberen Grenzwert der Funktion zu gering, so daß eine Extrapolation über den Meßbereich hinaus zumeist nicht möglich ist. In vielen Fällen exrechnet sich der Maximalwert sogar - wie bei den Meßreihen von Siliqua patula - niedriger als der höchste Meßwert, was biologisch wenig sinnvoll erscheint.

Die Reziprokfunktion erscheint demgegenüber als günstigere Lösung, da sie auch bei Kurven mit Wendepunkt gute Näherungen erreicht und der obere Grenzwert einen hinreichenden Abstand von den höchsten Meßwerten aufweist. Der Wendepunkt für die linearen Werte errechnet sich aus dieser Funktion aber zu einem zu frühen Zeitpunkt. Also stellt auch die Reziprokfunktion keine exakte Beschreibung von Wachstumskurven dar.

Die Pütter-Bertalanffy-Funktion enthält ebenso wie die Gompertz-Funktion den Zeitwert als negativen Exponenten von e. Nun ist:

$$
\mathrm{e}^{-x}=\frac{1}{\mathrm{e}^{x}}
$$

Formen wir in diesem Sinn die Bertalanffy-Funktion um, so schreibt sich Gl. (1):

$$
y \chi=Y_{\infty}-\frac{Y_{\infty}-Y_{0}}{\mathrm{e}^{K_{\chi}}}
$$

Die einmal logarithmierte Gompertz-Funktion Gl. (6) nimmt dann die Form an:

$$
\ln y=\ln Y_{\infty}-\frac{\ln Y_{\infty}-\ln Y_{0}}{\mathrm{e}^{\mathrm{C}_{\chi}}}
$$

Für die logarithmierte Reziprokfunktion ergab sich Gl. (13):

$$
\ln y=\ln Y_{\max }-\frac{\ln N}{\chi+\xi}
$$

Diese Darstellung der drei berücksichtigten Funktionen macht ihre grundsätzlich übereinstimmende Struktur deutlich. Die jeweilige Größe ergibt sich aus der Differenz zwischen dem oberen Grenzwert der Funktion und dem Quotienten eines konstanten Parameters im Zähler und dem variablen Zeitwert im Nenner. Der reziproke Zeitwert beschreibt die mit zunehmendem Alter sich asymptotisch dem Wert Null nähernden Wachstumsraten. Der Zeitwert wird bei der Reziprokfunktion von einem linearen Wert gebildet, bei der Gompertz- und der Bertalanffy-Funktion durch eine Potenz von e. Hierin liegt der wesentliche Unterschied der Funktionen.

Die Gompertz- und die Reziprok-Funktion beruhen auf logarithmischer Basis. Diese relative Darstellung entspricht mehr dem Wesen von Wachstumsvorgängen als die lineare Wiedergabe durch die Bertalanffy-Funktion, da die Zuwachsraten abhängig sind von der gegebenen Größe eines Organismus. 
Konstante relative Wachstumsraten würden bei semilogarithmischer Darstellung eine Gerade ergeben. Die in der Krümmung der logarithmischen Wachstumskurve zum Ausdruck kommende kontinuierliche Abnahme der relativen Wachstumsraten macht das Auftreten eines Wendepunktes bei der linearen Darstellung der gleichen Werte verständlich. Im Beginn der Entwicklung - besonders deutlich beim Embryo sind die linearen Zuwachsraten sehr gering, die auf die Ausgangsgröße bezogenen relativen Wachstumsraten aber um ein Vielfaches größer als beim späteren Wachstum. Im weiteren Verlauf vermindern sich die relativen Wachstumsraten fortlaufend, während der lineare Zuwachs mit zunehmender Größe des Individuums zunächst zunimmt, bis am Wendepunkt die zunehmenden linearen und abnehmenden relativen Wachstumsraten gleich groß werden und sich überschneiden. Danach nehmen mit zunehmendem Alter beide Wachstumsraten fortlaufend $a b$ und nähern sich dem Wert Null. Während sich die relative Wachstumskurve fortlaufend abflacht, beginnt und endet die absolute Wachstumskurve mit sehr geringen Steigungen und dazwischen liegt um den Wendepunkt der steilste Anstieg der Kurve. Die Auftragung der linearen Zuwachsraten als Funktion des Alters liefert eine schiefe Glockenkurve mit Maximum beim Wendepunkt.

Relative Wachstumsdarstellungen mit abklingenden Wachstumsraten liefern uns also eine rein mathematische, von Hypothesen unbelastete Deutung für das Auftreten eines Wendepunktes, wie schon Weymouth (1931) erkannte: "the inflection becomes a mere mathematical consequence of the course of relative growth." Jede zur x-Achse gekrümmte logarithmische Kurve ergibt unabhängig von der zugrunde liegenden Funktion für ihre linearen Werte einen Wendepunkt.

Eine zutreffende Angabe des Wendepunktes setzt aber die Kenntnis der genauen Formulierung des Kurvenverlaufs voraus, und diese Bedingung wird weder von der Reziprok- noch von der Gompertz-Funktion erfüllt. Die Ursache dürfte in einer unzutreffenden Beschreibung des Abklingens der Wachstumsraten liegen. Im Augenblick kann ich aber noch keine anderen Lösungen erkennen. Das Alter als Potenz von e bewährt sich anscheinend gut in der Gompertz-Funktion für den Anfangsteil der Kurven, aber weniger für den Abschnitt nach dem Wendepunkt. Das dürfte daran liegen, daß für die höheren Alterswerte diese Potenzen zu schnell ansteigen und den Quotienten sehr niedrigen Werten nähern, was wiederum zur Folge hat, daß der Maximalwert früher erreicht wird, als mit biologischen Gegebenheiten vereinbar ist.

Auch wenn die hier geprüften Funktionen keine ganz exakte Wiedergabe von Wachstumsdaten liefern, dürfte die von ihnen erreichte Näherung für viele Fragestellungen ausreichend sein. Darüber hinaus erscheinen sie sehr bedeutungsvoll für ein tieferes Eindringen in das Verständnis von Wachstumsvorgängen. Das gilt allerdings weniger für die Pütter-Bertalanffy-Funktion, zu deren Gunsten auf ihre $\mathrm{Ab}$ leitung aus dem Anabolismus-Katabolismus-Konzept hingewiesen wird. Thre lineare Fassung muß man aber als weniger günstig ansehen.

Nun sind in dem Pütter-Bertalanffy-Ansatz:

$$
\frac{\mathrm{d} w}{\mathrm{~d} \chi}=\alpha \cdot w^{2 / 3}-\psi \cdot w
$$


Anabolismus $\left(\alpha \cdot w^{2 / 3}\right)$ und Katabolismus $(\varkappa \cdot w)$ in Form ihrer allometrischen Beziehung zum Gewicht benutzt. Das hier vorliegende Problem der Allometrie-Summation läßt sich, wie wir oben sahen, über die beiden relativen Funktionen lösen. Beide Funktionen stellen also gleichberechtigte Lösungen des Anabolismus-KatabolismusAnsatzes dar, besitzen aber in höherem Maß den Charakter von Wachstumsmodellen.

Auch wenn sie keine ganz idealen Lösungen darstellen, erfüllen sie doch eine Reihe von Forderungen, die an eine Wachstumsformel zu stellen sind. Besondere Bedeutung kommt ihrer relativen Darstellung zu. Die Neubildung lebender Substanz hängt von der vorhandenen Menge ab - große Organismen haben größere Zuwachsraten als kleinere - somit erfaßt schon die relative Darstellung ein wesentliches Merkmal organischen Wachstums. In ihrer logarithmischen Form erlauben ferner die beiden relativen Funktionen durch Ausschaltung des Alterswertes die Ableitung der allometrischen Beziehung zwischen zwei Dimensionen eines wachsenden Organismus, die an zahllosen Beispielen bestätigt wurde. Darüber hinaus ermöglichen beide Funktionen eine identische Lösung für das Problem der Summation mehrerer allometrisch wachsender Glieder zu einem Ganzen. Die Bedeutung der logarithmischen Darstellung für das Auftreten eines Wendepunktes in der linearen (absoluten) Wachstumskurve hatte ich weiter oben behandelt. Wie wir sahen, wird er verursacht durch die mit zunehmendem Alter abnehmende Wachstumsgeschwindigkeit. Hier stoßen wir in der Wachstumsfunktion auf das Problem des Alterns von Organismen, nämlich die Frage nach der Ursache der Verminderung der Wachstumsgeschwindigkeit.

In einer sehr verdienstvollen Dissertation hat Hoeppe (1959) aus der Literatur 100 Vorschläge für Wachstumsfunktionen zusammengestellt und kritisch besprochen. Die Zusammenstellung ist nicht vollständig, schließt zum Beispiel nicht die in dieser Arbeit ausgewertete Reziprokfunktion von Zucker \& Zucker (1941) ein. Die Gesamtzahl der bis heute veröffentlichten Vorschläge dürfte etwa doppelt so hoch sein. Zum Teil stellen sie allerdings nur Anpassungen an spezielle Beispiele dar. Eine wirklich brauchbare Funktion sollte aber doch einen weiteren Anwendungsbereich umfassen. Für die drei hier berücksichtigten Funktionen triff das zu, da die auf das Wachstum von Muscheln angewandten Funktionen an anderer Stelle für das Wachstum von Fischen und Säugetieren benutzt wurden. Hier treffen wir auf eine andere Erkenntnis, die uns nur durch mathematische Modelle vermittelt werden kann, daß nämlich den sehr verschiedenartig erscheinenden Wachstumskurven aus weiten Bereichen des Tierreiches einheitliche Gesetzmäßigkeiten zugrunde liegen.

Diese Einsicht können kaum die von manchen Autoren vorgeschlagenen Reihenentwicklungen verschiedener Form vermitteln, die sich mit einer reinen Simulation der Zahlenfolgen begnügen.

Besondere Schwierigkeiten hat die Darstellung des Wendepunktes in linearen Wachstumskurven bereitet. Auch wenn wir sein Auftreten aus den relativen Wachstumsbeziehungen erklären können, bildet die Beschreibung seiner exakten Lage immer noch ein offenes Problem. Da der Verlauf der sogenannten logistischen Funktion einen Wendepunkt einschließt, haben sie verschiedene Autoren zur Anwendung auf Wachstumsdaten vorgeschlagen. Die durch ihre Symmetrie um den Wendepunkt gekennzeichnete Funktion erscheint höchstens nach der Einführung weiterer Para- 
meter für die Beschreibung von Wachstumsdaten geeignet. In ähnlicher Weise hat Scharf $(1972,1973)$ neuerdings mit dem Tangens hyperbolicus und anderen SigmoidFunktionen versucht, den Wachstumsablauf menschlicher Embryonen zu simulieren. Solche Verfahren mögen geeignet sein, hinreichende Näherungen an gegebene Daten zu simulieren. Keine der besprochenen Funktionen liefert eine vollkommen befriedigende Simulation von Wachstumskurven, und es muß daher ein erstrebenswertes Ziel bleiben, vorhandene Mängel der heutigen Wachstumsfunktionen zu beheben. Dieses wäre vielleicht durch zusätzliche Parameter möglich. Allerdings übersehe ich noch nicht, an welcher Stelle und in welcher Form sie eingesetzt werden könnten.

Scharf (1977) hat neuerdings eine um einen Parameter erweiterte GompertzFunktion vorgeschlagen, aber nur an einem einzigen Beispiel - dem menschlichen Embryonalwachstum - getestet. Ob diesem Versuch eine allgemeinere Bedeutung zukommt, bleibt offen. Man muß immer im Auge behalten, daß eine reine Simulation von Zahlenfolgen nicht die Lösung eines Wachstumsmodelles darstellt.

Für die Aufstellung eines grundsätzlich anderen Wachstumsmodells lassen sich noch keine Ansätze erkennen. So werden wir uns auch weiterhin mit den gegebenen Näherungslösungen begnügen müssen.

Danksagungen. Zur Durchführung der erforderlichen Berechnungen diente mir ein KleinComputer 700 der Firma Wang, der mir dankenswerterweise von der Deutschen Forschungsgemeinschaft zur Verfügung gestellt war. Dank schulde ich ebenfalls dem Direktor der Biologischen Anstalt Helgoland, Herrn Prof. O. Kinne, der mir einen Arbeitsplatz in der Hamburger Zentrale zur Verfügung stellte. Herrn Prof. J. W. Hedgpeth danke ich für die Bestätigung der noch gültigen Benennung von Siliqua patula.

\section{ZITIERTE LITERAT'UR}

Bagenal, T. B., 1955. The growth rate of the long rough dab Hippoglossoides. J. mar. biol. Ass. U. K. 34, 297-311.

- 1955. The growth rate of the long rough dab Hippoglossoides platessoides. J. mar. biol. Ass. U. K. 34, 643-647.

Bertalanffy, L. von, 1934. Untersuchungen ïber die Gesetzlichkeit des Wachstums. 1. Allgemeine Grundlagen der Theorie. Wilhelm Roux Arch. EntwMech.Org. 131, 613-653.

Beverton, R. J. H. \& Holt, S. J., 1957. On the dynamics of exploited fish populations. Fishery Invest., Lond. (Ser. 2) 19, 1-533.

Dubois, E., 1897. Über die Abhängigkeit des Gehirngewichtes von der Körpergröße. Arch. Anthrop. 25, 1-28.

Ford, E., 1933. An account of the herring investigations conducted at Plymouth during the years from 1924-1933. J. mar. biol. Ass. U. K. 19, 305-384.

Gompertz, B., 1825. On the nature of the function expressive of the law of human mortality. Phil. Trans. R. Soc. 36, 513-585.

Hoeppe, K., 1959. Das reaktionskinetische Grundgesetz auf das Wachstum bezogen und anderen Wachstumsformulierungen gegenübergestellt. Diss., Univ. Gießen, 201 pp.

Hohendorf, K., 1966. Eine Diskussion der Bertalanffy-Funktion und ihre Anwendung zur Charakterisierung des Wachstums von Fischen. Kieler Meeresforsch. 22, 70-97.

Huxley, J. S. \& Teissier, G., 1936. Zur Terminologie des relativen Größenwachstums. Biol. Zbl. 56, 381-383.

Krüger, F., 1962. Über die mathematische Darstellung des tierischen Wachstums. Naturwissenschaften $49,454$. 
- 1964. Mathematische Ableitung der allometrischen Wachstumsfunktion. Zool.Anz. (Suppl.) 27, 249-253.

- 1965. Zur Mathematik tierischen Wachstums. I. Grundlagen einer neuen Wachstumsfunktion. Helgoländer wiss. Meeresunters. 12, 78-136.

- 1970. Zum Problem der Allometrie-Summation. Biol. Zbl. 89, 65-85.

- 1973. Zur Mathematik tierischen Wachstums. II. Vergleich einiger Wachstumsfunktionen. Helgoländer wiss. Meeresunters. 25, 509-550.

Pütter, A., 1920. Wachstumsähnlichkeiten. Pflügers Arch. ges. Physiol. 180, 298-340.

Scharf, J.-H., 1969. Zum Körperlängenwachstumsgesetz der menschlichen Leibesfrucht. Acta anat. $\mathbf{7 3}, 10-18$.

- 1977. Wachstum. Verh. anat. Ges., Jena 71, 29-58.

- Peil, J. \& Helwin, H., 1972. Systematische Untersuchungen zur eigentlich nichtlinearen Regression mit Sigmoidfunktionen. Teil I. Theoretische Einführung. Biometr. Z. 14, 387-397.

- Peil, J. \& Helwin, H., 1973. Systematische Untersuchungen zur eigentlich nichtlinearen Regression mit Sigmoidfunktionen. Fortsetzung und Schluß. Biometr. Z. 21-46; 179-189.

- Peil, J., 1975. Ein Algorithmus zur Wertebestimmung der Parameter in der Gompertzschen Wachstumsfunktion. Gegenbaurs morph. Jb. 121, 389-420.

Sella, M., 1929. Migrazioni e habitat del tonno (Thunnus thynnus) studiato col metodo degli ami, con osservazioni su l'ascrescimento. Memorie R. Com. talassogr. ital. 156, 1-24.

Smith, C. A., 1954. Biomathematics. Griffin, London.

Snell, O., 1891. Die Abhängigkeit des Gehirngewichtes von dem Körpergewicht und den geistigen Fähigkeiten. Arch. Psychiat. NervKrankh. 23, 436-446.

Thompson, D'Arey W., 1952. On growth and form. Univ. Press, Cambridge 1, 1-464.

Walford, L. A., 1946. A new graphic method of describing the growth of animals. Biol. Bull. mar. biol. Lab., Woods Hole 90, 141-147.

Weymouth, F. W., McMillin, H. C., 1930. The relative growth and mortality of the Pacific razor clam (Siliqua patula), and their bearing on the commercial fishery. Bull. U.S. Fish Commn 46, 543-567.

- - Rich, W. H., 1931. Latitude and relative growth in the razor clam Siliqua patula. J. exp. Biol. 8, 228-249.

Zucker, L. \& Zucker, T. F., 1941. A simple time weight relation observed in well nourished rats. J. gen. Physiol. 25, 445-463. 